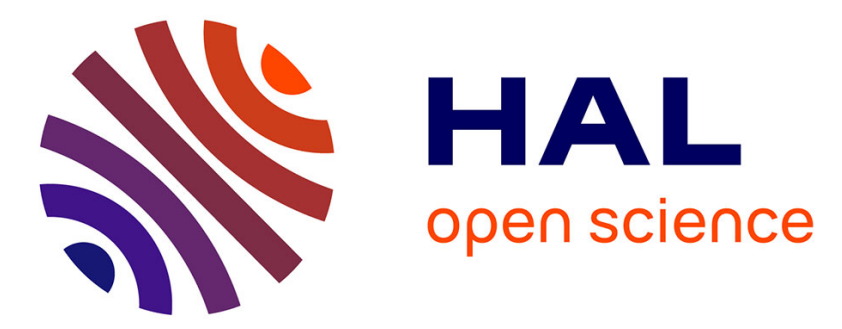

\title{
Are More Risk-Averse Agents More Optimistic? Insights from a Simple Rational Expectations Equilibrium Model
} Elyès Jouini, Clotilde Napp

\section{To cite this version:}

Elyès Jouini, Clotilde Napp. Are More Risk-Averse Agents More Optimistic? Insights from a Simple Rational Expectations Equilibrium Model. Economics Letters, 2008, 101, pp.73-76. halshs00176630v2

\section{HAL Id: halshs-00176630 \\ https://shs.hal.science/halshs-00176630v2}

Submitted on 2 Jun 2010

HAL is a multi-disciplinary open access archive for the deposit and dissemination of scientific research documents, whether they are published or not. The documents may come from teaching and research institutions in France or abroad, or from public or private research centers.
L'archive ouverte pluridisciplinaire HAL, est destinée au dépôt et à la diffusion de documents scientifiques de niveau recherche, publiés ou non, émanant des établissements d'enseignement et de recherche français ou étrangers, des laboratoires publics ou privés. 


\title{
Are more risk-averse agents more optimistic? Insights from a simple rational expectations equilibrium model.
}

\author{
November 3, 2006
}

\begin{abstract}
We analyze the link between pessimism and risk-aversion.. We consider a model of partially revealing, competitive rational expectations equilibrium with diverse information, in which the distribution of risk-aversion across individuals is unknown. We show that when a high individual level of risk-aversion is taken as a signal for a high average level of risk-aversion, more risk-averse agents are more optimistic. This correlation between individual risk-aversion and optimism leads to a pessimistic "consensus belief" hence to an increase in the market price of risk. Risk-sharing schemes and welfare implications are analyzed. We show that agents' welfare may increase upon the receipt of more precise information.
\end{abstract}

JEL Numbers: G12; D53; D82

Keywords: Optimism; risk-aversion; rational expectations; risk-premium; heterogenous beliefs 


\section{Introduction}

Several papers in recent financial economics literature study models in which agents have heterogenous beliefs. Beliefs heterogeneity might result from differential information (Green, 1973, Grossman, 1976, Genotte-Leland, 1980, Hellwig, 1980, Grossman-Stiglitz, 1981, Diamond-Verrecchia, 1981, Kyle, 1989), different opinions (Varian, 1985, 1989, Abel, 1989, Harris-Raviv, 1993), different priors and Bayesian updating (Williams, 1977, Detemple-Murthy, 1994, Zapatero, 1998, Gallmeyer, 2000, Basak, 2000, GallmeyerHollifield, 2002), different processing of information (Dumas et al., 2005, ScheinkmanXiong, 2003), behavioral biases (Brunnermeier-Parker, 2005, Gollier-Muermann, 2006).

In equilibrium models with beliefs heterogeneity, it is shown that the belief of the representative agent is given by the average of the individual beliefs weighted by the individual risk-tolerances (Abel, 1989, Detemple-Murthy, 1994, Calvet et al., 2002, JouiniNapp, 2004, 2006). This means that in order to determine the impact of beliefs heterogeneity on the equilibrium characteristics, it is particularly important to investigate the nature of the link between individual risk-aversion and beliefs.

In this paper, our goal is to analyze the nature of this link in a rational expectations equilibrium framework.

For this purpose, we consider a model of partially revealing, competitive rational expectations equilibrium with diverse information. More precisely, we analyze a "large" market with a continuum of traders who posess diverse pieces of private information about the risky asset's return as in e.g. Grossman (1976), Hellwig (1980), GenotteLeland (1980), Diamond-Verrecchia (1981), Admati (1985), Kyle (1989). Contrarily to standard models, we suppose that agents do not know the distribution of risk-aversion in the economy. Private information is aggregated and, due to the noise on the distribution 
of risk-aversion, is only partially revealed by the equilibrium prices. The equilibrium prices together with the private information, create the beliefs held by agents in equilibrium.

Assuming an imperfect knowledge of the risk-aversion is not an unrealistic assumption since the individual (or collective) risk-aversion is in general hard to estimate and the estimations strongly depend on the chosen methodology (lotteries as in Donkers et al, 2001, option prices as in Jackwerth, 2000, etc.). As underlined by Jackwerth (2000), "estimating risk-aversion functions directly is still notoriously difficult". As a consequence, there is no consensus in the literature on a precise aggregate risk-aversion level. As underlined by Brennan and Torous (1999) "there is considerable controversy among economists about what constitutes a reasonable level of risk aversion". Furthermore, many recent models consider state dependent preferences (rank-dependent utility, relative consumption utility, habit formation, recursive utility) hence state dependent risk-aversion levels; the knowledge of the individual level of risk-aversion would suppose a continuous time monitoring of the individual choices and risk attitudes.

We prove existence results for such rational expectations equilibria and we obtain closed form solutions for linear equilibria, thereby providing a theoretical rational expectations framework for the study of the link between individual belief and risk-aversion. The question under consideration is the following. Are more risk-averse agents more optimistic? More optimistic refers to a higher posterior (after the observation of the private signal and the equilibrium price) expected return of the risky asset. We start from a model in which individual risk-aversions and individual beliefs are independent. We suppose that a low (resp. high) individual level of risk-aversion is taken as a private signal for a low (resp. high) average level of risk-aversion. We show that a positive correlation between optimism and risk-aversion naturally emerges. The intuition is as 
follows. For a given equilibrium price, which, as we shall see, increases with the return of the risky asset and decreases with the average level of risk-aversion, a more risk-averse agent perceives a higher average level of risk-aversion and, by observing a given equilibrium price, infers a higher expected return. Furthermore we obtain that the intensity of this correlation depends on the dispersion of the individual levels of risk-aversion as well as on the degree at which the individual relies on his own level of risk-aversion to estimate the average level of risk-aversion. In particular, when the average risk-aversion is exogenously specified, i.e., when the agents do not consider their own level of risk-aversion as informative for the average level of risk-aversion, there is no correlation between optimism and risk-aversion.

When this correlation is positive, we analyze the consequences in terms of market price of risk, risk-sharing and welfare. We find that the market price of risk is higher than in the standard framework, which is interesting in light of the risk premium puzzle. The interpretation is the following. Since the belief of the representative agent is an average of the individual beliefs weighted by the risk-tolerances, the positive correlation between optimism (resp. pessimism) and risk-aversion (resp. risk-tolerance) induces a more pessimistic consensus belief even though there is no bias on average on the individual prior beliefs; pessimism at the aggregate level induces a higher risk premium and a higher market price of risk. The reason why pessimism increases the market price of risk is not that a pessimistic representative agent requires a higher market price of risk. He/She requires the same market price of risk but his/her pessimism leads him/her to underestimate the average rate of return of the risky asset. Thus the objective expectation of the equilibrium market price of risk is greater than the representative agent's subjective expectation, hence is greater than the standard market price of risk (see Abel, 2002, and Jouini-Napp, 2006). 
We also analyze the extent to which risk-sharing schemes are modified. In the classical situation, on average over the signals, a relatively very risk-averse (resp. tolerant) individual will have very low (resp. high) optimal demand. In our setting, due to the positive correlation between optimism and risk-aversion, the very risk-averse becomes more optimistic, which heightens his optimal demand, and the very risk-tolerant agent becomes more pessimistic, which lowers his optimal demand. In the end, there is less dispersion in the optimal demands and less risk-sharing.

Our model also makes it possible to analyze how the arrival of more precise information affects the agents' utility at the individual or collective level. The correlation between optimism and risk-aversion limits risk-sharing and has then a negative impact on welfare. The arrival of more precise information therefore has a double impact: it weakens the adverse effect on trade (as risk-taking agents become less pessimistic, they offer more insurance) and at the same time it strenghtens the Hirschleifer effect (agents are no longer able to insure against news that has already arrived). The first effect fosters risk-sharing trade, while the second one discourages it. The paper discusses a situation where the positive effect on trade offsets the negative effect. This means that agents welfare may increase upon the receipt of more precise information. Although the result seems intuitive, most of the previous literature has focused on the case with homogenous beliefs. In such a framework, only the Hirschleifer effect is at work, therefore better information typically reduces welfare.

The paper is organized as follows. Section 2 presents the model and the equilibrium concept. In Section 3, existence results as well as closed-form solutions in specific settings are provided. Section 4 analyzes the correlation between risk-aversion and optimism as well as other properties of the equilibria under consideration. All proofs are in the Appendix. 


\section{The model}

We shall use the following version of Admati (1985)'s model. There is a continuum of agents, indexed by $i \in[0,1]$ and 2 periods denoted by 0 and 1 . Agents trade at date 0 and consume at date 1. Each agent $i$ is endowed with an initial wealth $w_{0 i}$ and one unit of a given risky asset, and can allocate his initial endowment between a riskless asset and the risky asset. For each unit purchased at date 0, the riskless asset yields one unit and the risky asset $\widetilde{X}$ units of a single consumption good at date 1. Using the riskless asset as numeraire, let $p$ be the price of the risky asset. If agent $i$ holds $\theta_{i}$ units of the risky asset, his wealth at date 1 is given by $\widetilde{w_{1 i}}=w_{0 i}+\theta_{i} \tilde{X}-\left(\theta_{i}-1\right) p$. Each agent $i$ maximizes his expected utility from consumption $E_{i}\left[u_{i}\left(\widetilde{w_{1 i}}\right)\right]$. We suppose that the utility functions exhibit constant absolute risk-aversion, more precisely $u_{i}(w)=-\exp -a_{i} w$ where the measurable function $\widetilde{a}:[0,1] \rightarrow \mathbb{R}_{+}$is such that $a_{i}$ represents agent $i$ 's coefficient of risk-aversion. The distribution of risk-aversion among agents is unknown; each agent only knows his own level of risk-aversion. In standard rational expectations models, the unique meaningful risk-aversion parameter at the equilibrium is the representative agent risk-aversion, which is given by the harmonic average of the individual levels of

risk-aversion. We denote by $\rho \equiv \int_{0}^{1} \frac{1}{a_{i}} d i$ the average risk-tolerance and by $\widetilde{Z} \equiv \rho^{-1}$ the (harmonic) average risk-aversion. We suppose that both $\widetilde{X}$ and $\widetilde{Z}$ are unknown and stochastic and that all agents have the same prior distribution for $(\widetilde{X}, \widetilde{Z})$. Under the assumption of CARA utility function, agent $i$ 's demand for the risky asset is independent of his initial wealth. It depends only upon the price $p$ and the expectation operator $E_{i}$, which is determined by agent $i$ 's information $I_{i}$. The information $I_{i}$ of agent $i$ consists of the equilibrium price and of his private information. As in standard models, agent $i$ 's private information first consists of the observation of a signal $\tilde{Y}_{i}$ which is 
correlated with $\widetilde{X}$. More specifically, $\widetilde{X} \sim \mathcal{N}\left(m_{x}, \sigma_{x}^{2}\right)$, the private signal $\tilde{Y}_{i}$ is given by $\widetilde{Y}_{i}=\widetilde{Y}+\widetilde{\varepsilon_{i}}$, where $\widetilde{\varepsilon_{i}} \sim \mathcal{N}\left(0, \sigma_{\varepsilon}^{2}\right), \widetilde{Y}=\widetilde{X}-\widetilde{U}, \tilde{U} \sim \mathcal{N}\left(0, \sigma_{u}^{2}\right), \widetilde{\varepsilon_{i}}$ and $\widetilde{Y}$ are independent, and $\widetilde{\varepsilon_{i}}$ is independent of $\widetilde{\varepsilon_{j}}, j \neq i$. The random variable $\widetilde{Y}_{i}$ is a perturbation of the random payoff $\widetilde{X}$ by an error term $\widetilde{U}$ common to all agents and by $\widetilde{\varepsilon_{i}}$, which affects only agent $i$ 's signal. We assume, as in Admati (1985) a "law of large numbers", i.e., that ${ }^{1} \int_{0}^{1} \widetilde{\varepsilon}_{i} d i=0$. Note that this model can account for diverse information as well as diverse opinion. Indeed, it is equivalent to assume that each agent has a signal and that the signals are randomly drawn around the true return or that each agent has an opinion (this opinion is modeled by a belief conditional to the observation of a private signal) and that these opinions are also randomly drawn around the true return. In the differential information framework individuals care about other's beliefs because they contain information (others private signals) and in particular, the average of the individual signals contains more information than each individual signal. In the heterogenous opinions framework, individuals care about other's opinions if we assume that everyone thinks that the average opinion is closer to the truth than any individual opinion (conformity, informational social influence).

Moreover, unlike previous literature, we suppose that the distribution of risk-aversion in the economy is unknown and the average level of risk aversion is modeled by a random variable. This randomness can be interpreted in two ways leading to two possible specifications. The first interpretation is that there are econometric estimations of the

\footnotetext{
${ }^{1}$ In general, if $\left(X_{i}\right)_{i \in[0,1]}$ is a process of independent variables such that $E\left[X_{i}\right]=0$ for all $i$ and $\operatorname{Var}\left[X_{i}\right]$ is uniformly bounded, then for every sequence $\left\{i_{n}\right\}$ of distinct indices from $[0,1]$, the strong law of large numbers applied to the sequence $\left(X_{i_{n}}\right)_{n \in N}$ yields that $\frac{1}{N} \sum X_{i_{n}} \rightarrow 0$ a.s. Thus it seems natural to define $\int_{0}^{1} X_{i} d i \equiv 0$. We will adopt the slightly more general convention: if the $\left(X_{i}\right)_{i \in[0,1]}$ are independent, with mean zero and bounded variance, and $X_{i}^{\prime}$ is almost surely integrable then $\int_{0}^{1}\left(X_{i}+\right.$ $\left.X_{i}^{\prime}\right) d i \equiv \int_{0}^{1} X_{i}^{\prime} d i$. In our context, this means that $\int_{0}^{1} \widetilde{Y}_{i} d i=\widetilde{Y}$
} 
average level of risk-aversion that are available and common knowledge. We suppose then that agents agree on an exogenous distribution ${ }^{2}$ of $\widetilde{Z} \sim \mathcal{N}\left(m_{z}, \sigma_{z}^{2}\right)$ where $\sigma_{z}^{2}$ measures the confidence level of these estimations. In this case, agents private information only consists of the private signal since agents do not infer any information from their own observed level of risk-aversion. The second interpretation is that individual and collective risk-aversion levels are difficult to estimate in particular because these parameters may be state dependent and may evolve through time. In that case, agents rely on their own level of risk-aversion in order to estimate the average level of risk-aversion. In this setting, agents interpret their own level of risk-aversion as private information on the average level of risk-aversion. We do not specify a particular form for the agents prior on the risk-aversion distribution and the learning process. We simply assume that the posterior distribution of the harmonic mean $\widetilde{Z}$ following the observation of the individual level of risk-aversion $a_{i}$ satisfies $\widetilde{Z} \mid a_{i} \sim \mathcal{N}\left(A_{i}, \sigma_{z}^{2}\right)$ where $A_{i}=k a_{i}-\ell$ with $\ell \geq 0$ and $k \in[0,1]$. The normality condition on the posterior distribution of $\widetilde{Z}$ is particularly satisfied if we assume for example that agents have a prior distribution of risk-tolerance $\frac{1}{\tilde{a}} \sim \mathcal{N}\left(\frac{1}{z}, \frac{\sigma^{2}}{z^{2}}\right) \operatorname{with}^{3} z \sim \mathcal{N}\left(m, \tau^{2}\right)$. However, in this case, the variance of the posterior distribution depends on $a_{i}$, and for reasons of tractability we impose in

${ }^{2}$ In fact, $\widetilde{Z}$ can only be positive, but it is standard in financial economics, for easily understandable reasons of tractability, to suppose a normal distribution, with a "reasonably small" variance.

${ }^{3}$ We obtain that the posterior distribution of $Z$, conditional to the observation of $a_{i}$, is normal with mean $\frac{\frac{a_{i}}{a_{i}^{2} \sigma^{2}}+\frac{m}{\tau^{2}}}{a_{i}^{2} \sigma^{2}}+\frac{1}{\tau^{2}}$ and variance $\left(\frac{1}{a_{i}^{2} \sigma^{2}}+\frac{1}{\tau^{2}}\right)^{-1}$. In particular, for $\tau=\infty$, which corresponds to the situation where there is no prior on $Z$, the posterior distribution of $Z$, is normal with mean $a_{i}$ and variance $a_{i}^{2} \sigma^{2}$.

More generally, if the agents have priors on the risk aversion distribution that are symmetric with respect to a given parameter $\mu$ (and then centered on $\mu$ ) and flat priors on $\mu$, the observation of their own level of risk aversion $a_{i}$ would lead to posteriors on the risk aversion distribution that are centered on $a_{i}$. 
our model that the variance be the same for all agents. In fact, everything works as if agents had another characteristic denoted by $A_{i}$ that is directly linked to $a_{i}$ and that is such that the harmonic average of the $a_{i}$ is equal to the arithmetic average of the $A_{i}$. Now, the justification for letting the mean $A_{i}$ be of the form $k a_{i}-\ell$ is the following. For a large class of distributions, including the distributions that are symmetric with respect to their average, without further information about the distribution of the level of risk-aversion among individuals, the agent's own level of risk-aversion $a_{i}$ is the best possible estimate of the arithmetic average level of risk-aversion. However, the agent's goal is to estimate the harmonic mean of risk-aversion and it is well known that the harmonic mean systematically lies below the arithmetic mean. The factor $k$ measures the degree at which individuals rely on their own level of risk aversion in order to estimate the average level of risk aversion (idiosyncratic part) and $-\ell$ corresponds to the isosyncratic part of the estimation.

We suppose that the objective distribution of risk-aversion in the economy as well as the subjective posterior belief $\widetilde{Z} \mid a_{i}$ are independent of $\widetilde{X}, \widetilde{U}$ and $\widetilde{\varepsilon_{i}}$ and that all the introduced random variables have a jointly normal distribution.

Since in the large economy, each agent is "small" and the private signals are independently distributed, the particular realizations of these private signals should have no effect on the realized equilibrium price. We will therefore search for equilibria in this economy in which the equilibrium price only depends on "market aggregates" $\widetilde{Y}$ and $\widetilde{Z}$. The following definition is standard.

Definition 1. An equilibrium for the economy is defined by a price $\widetilde{p}$ and demand Analogously, we obtain $z \sim \ln \mathcal{N}\left(\frac{\frac{\ln a_{i}}{\sigma^{2}}+\frac{m}{\tau^{2}}}{\frac{1}{\sigma^{2}}+\frac{1}{\tau^{2}}}-\frac{1}{2} \sigma^{2}, \frac{1}{\frac{1}{\sigma^{2}}+\frac{1}{\tau^{2}}}\right)$ (that we may approximate by a normal distribution with the same first two moments) when $\widetilde{a} \sim \ln \mathcal{N}\left(\mu, \sigma^{2}\right)$ with $\mu \sim \mathcal{N}\left(m, \tau^{2}\right)$. 
functions $\left(\widetilde{\theta_{i}}\right)_{i \in[0,1]}$ such that (a) $\widetilde{p}$ is $(\widetilde{Y}, \widetilde{Z})$ measurable; (b) $\widetilde{\theta_{i}} \in \arg \max E_{i}\left[u_{i}\left(\widetilde{w_{1 i}}\right)\right]$; (c) $\int_{0}^{1} \widetilde{\theta}_{i} d i=1$.

In the next section, an equilibrium in the class of linear functions of $\widetilde{Y}$ and $\widetilde{Z}$ is sought.

Let us recall the properties of two standard models that we will use as benchmarks for our analysis: the Walrasian equilibrium model (without rational expectations) and the rational expectations model with perfect knowledge of the risk-aversion distribution.

In the Walrasian setting, the information $I_{i}$ of agent $i$ only consists of the observation of the signal $\tilde{Y}_{i}$ so that the conditional distribution of $\tilde{X}$ given $I_{i}$ is given by $\mathcal{N}\left(m_{x}+\right.$ $\left.\left(y_{i}-m_{x}\right) \frac{\sigma_{y}^{2}}{\sigma_{\varepsilon}^{2}+\sigma_{y}^{2}}, \sigma_{u}^{2}+\frac{\sigma_{\varepsilon}^{2} \sigma_{y}^{2}}{\sigma_{\varepsilon}^{2}+\sigma_{y}^{2}}\right)$. The optimal demand of agent $i$ is given by

$$
\theta_{i}=\frac{1}{a_{i}} \frac{m_{x}+\left(\tilde{Y}_{i}-m_{x}\right) \frac{\sigma_{y}^{2}}{\sigma_{\varepsilon}^{2}+\sigma_{y}^{2}}-\tilde{p}}{\sigma_{u}^{2}+\frac{\sigma_{\varepsilon}^{2} \sigma_{y}^{2}}{\sigma_{\varepsilon}^{2}+\sigma_{y}^{2}}} .
$$

We then derive from the market clearing condition that

$$
\begin{aligned}
\tilde{p} & =m_{x}+\left(\widetilde{Y}-m_{x}\right) \frac{\sigma_{y}^{2}}{\sigma_{\varepsilon}^{2}+\sigma_{y}^{2}}-z\left(\sigma_{u}^{2}+\frac{\sigma_{\varepsilon}^{2} \sigma_{y}^{2}}{\sigma_{\varepsilon}^{2}+\sigma_{y}^{2}}\right) \\
& =\left(1-\beta_{W}\right) m_{x}+\beta_{W} \tilde{Y}-z V_{W}
\end{aligned}
$$

where $\beta_{W}$ is equal to $\frac{\sigma_{y}^{2}}{\sigma_{\varepsilon}^{2}+\sigma_{y}^{2}}$ and $V_{W}$ is the ex-post variance $\operatorname{Var}\left(X \mid I_{i}\right)$.

In the rational expectations setting with perfect knowledge of the risk-aversion distribution, the agent learns from the observation of $\tilde{Y}_{i}$ and the equilibrium price. The equilibrium price is given by ${ }^{4}$

\footnotetext{
${ }^{4}$ It is well known that this model leads to a fully revealing equilibrium and then becomes problematic. Nevertheless, we present it as a benchmark.
} 


$$
\begin{aligned}
\tilde{p} & =\tilde{Y}-z \sigma_{u}^{2} \\
& =\left(1-\beta_{R}\right) m_{x}+\beta_{R} \tilde{Y}-z V_{R}
\end{aligned}
$$

where $\beta_{R}=1$ and $V_{R}$ is the ex-post variance $\operatorname{Var}\left(X \mid I_{i}\right)$.

Everything works as in a Walrasian setting where all agents would share their information and would then have a perfect knowledge of $\tilde{Y}=\tilde{X}-\tilde{U}$. Equation (2.5) is then a particular case of Equation (2.3) and corresponds to $\sigma_{\varepsilon}^{2}=0$. It is immediate through these equations to see that the harmonic average $z$ of the individual levels of risk-aversion is the only meaningful risk-aversion parameter at the equilibrium. It summarizes all the useful information about the risk-aversion distribution and corresponds to the risk-aversion level of the representative agent. It also measures the sensitivity of the equilibrium price with respect to the posterior level of risk $\operatorname{Var}\left(X \mid I_{i}\right)$.

\section{Existence of equilibria}

To begin, we consider the case of an exogenous specification of the average level of risk-aversion and then the more interesting case of an endogenous specification.

\subsection{Exogenous specification of the average risk-aversion}

In this section, we suppose that agents agree on an exogenous distribution of $\widetilde{Z} \sim$ $\mathcal{N}\left(m_{z}, \sigma_{z}^{2}\right)$ and do not infer any information from their own level of risk-aversion. Moreover, for the simplicity of the results in this setting, we assume that $\sigma_{u}=0$.

Theorem 1. If $\widetilde{Z} \sim \mathcal{N}\left(m_{z}, \sigma_{z}^{2}\right)$ and $\sigma_{u}=0$, then there exists a unique equilibrium 
price within the class of functions of the form

$$
\widetilde{p}=\alpha_{e}+\beta_{e} \tilde{Y}-\gamma_{e} \widetilde{Z}
$$

with $\gamma_{e} \neq 0$

This price has

$$
\left\{\begin{array}{c}
\alpha_{e}=\left(1-\beta_{e}\right) m_{x}+\left(\beta_{e} \sigma_{\varepsilon}^{2}-\left(1-\beta_{e}\right) \sigma_{x}^{2}\right) m_{z} \\
\beta_{e}=\frac{\sigma_{x}^{2}+\sigma_{x}^{2} \sigma_{\varepsilon}^{2} \sigma_{z}^{2}}{\sigma_{x}^{2}+\sigma_{x}^{2} \sigma_{\varepsilon}^{2} \sigma_{z}^{2}+\sigma_{\varepsilon}^{4} \sigma_{z}^{2}}=\frac{1+\sigma_{\varepsilon}^{2} \sigma_{z}^{2}}{1+\sigma_{\varepsilon}^{2} \sigma_{z}^{2}+\frac{\sigma_{\varepsilon}^{4}}{\sigma_{x}^{2}} \sigma_{z}^{2}} \\
\gamma_{e}=\beta_{e} \sigma_{\varepsilon}^{2}
\end{array}\right.
$$

Another way to write the equilibrium price is

$$
\widetilde{p}=\left(1-\beta_{e}\right) m_{x}+\beta_{e} \widetilde{Y}-\gamma_{e}\left(\widetilde{Z}-m_{z}\right)-V_{e} m_{z}
$$

where $V_{e}$ is the ex-post variance $\operatorname{Var}\left(X \mid I_{i}\right)$. Note that this variance does not involve the signal value. It is known ex-ante and is common to all the individuals. The price involves then a convex combination of $\widetilde{X}$ and its average $m_{x}$ and $\beta_{e}$ measures the weight put on $\widetilde{X}$. We check that, as expected, $\beta_{e}$ decreases with $\sigma_{\varepsilon}^{2}$. Furthermore, when $\sigma_{z}^{2} \rightarrow \infty$, the price becomes uninformative and $\beta_{e}$ converges to $\frac{\sigma_{x}^{2}}{\sigma_{x}^{2}+\sigma_{\varepsilon}^{2}}$ which corresponds, as expected, to the weight $\beta_{W}$ of $\tilde{X}$ in the Walrasian equilibrium. Finally, $\beta_{e}$ decreases when $\sigma_{x}^{2}$ increases and $\frac{\sigma_{x}^{2}}{\sigma_{\varepsilon}^{2}}$ is kept constant (constant relative precision of the signal) which is also natural. 


\subsection{Endogenous specification of the average risk-aversion}

We now consider the setting with an endogenous specification of the average level of risk-aversion. In this setting, agents interpret their own level of risk-aversion as private information on the average level of risk-aversion. We simply assume that the posterior

distribution of the harmonic mean $\widetilde{Z}$ following the observation of the individual level of risk-aversion $a_{i}$ satisfies $\widetilde{Z} \mid a_{i} \sim \mathcal{N}\left(A_{i}, \sigma_{z}^{2}\right)$ where $A_{i}=k a_{i}-\ell$ with $\ell \geq 0$ and $k \in[0,1]$.

\subsubsection{Existence of an equilibrium}

We start by proving the existence of an equilibrium in the general case with $\sigma_{u}>0$ and then consider the case with $\sigma_{u}=0$.

Theorem 2. For $\sigma_{u}>0$, there exists an equilibrium price within the class of functions of the form

$$
\widetilde{p}=\alpha+\beta \widetilde{Y}-\gamma \widetilde{Z}
$$

with $\gamma>0$ and $\beta>0$.

The equilibrium price for the risky asset is an increasing function of $\widetilde{X}$, its payoff, and a decreasing function of $\widetilde{Z}$, the average level of risk-aversion. When the price of the risky asset increases, an individual is uncertain whether it is the result of an increase in the risky asset payoff or whether, on average, everyone is less risk-averse. The observation of a given price should lead to an update of the distributions of $\widetilde{X}$ and $\widetilde{Z}$ in two opposite directions. 
Explicit computations lead to

$$
\widetilde{p}=(1-\beta) m_{x}+\beta \widetilde{Y}-\frac{1}{(k-1) D+1} V \widetilde{Z}-\frac{D}{(k-1) D+1} V \ell
$$

where $V$ is the posterior variance and where $\beta$ and $D$ are given parameters between 0 and 1. In fact, this price corresponds to the equilibrium price in a Walrasian setting where agent $i$ has a belief on $\tilde{X}$ given by $\tilde{X} \mid I_{i} \sim \mathcal{N}\left(E_{i}(\tilde{X}), V\right)$ where $E_{i}(\tilde{X})=E\left[\tilde{X} \mid I_{i}\right]$. It can be written in the form,

$$
\widetilde{p}=\frac{\int \frac{E_{i}(\tilde{X})}{a_{i}} d i}{\int \frac{1}{a_{i}} d i}-V z
$$

and corresponds to the equilibrium price in an economy with homogenous beliefs in which the common belief would be given by the average of the individual beliefs weighted by the individual levels of risk-tolerance.

In order to obtain explicit solutions and to analyze qualitative properties of the equilibria, we now consider the case $\sigma_{u}=0$. We suppose that there is enough noise in the economy, more precisely we suppose that $\sigma_{\varepsilon}^{2} \sigma_{z}^{2} \geq 4 k$. This condition is analogous to those in Glosten (1989) or Bhattacharya and Spiegel (1992). In this case, we obtain two possible linear rational expectations equilibria and we shall see that one is more natural than the other.

Theorem 3. If $\sigma_{u}=0$, and if $\sigma_{\varepsilon}^{2} \sigma_{z}^{2} \geq 4 k$, then there exist two possible equilibrium prices within the class of functions of the form

$$
\widetilde{p}=\alpha+\beta \widetilde{X}-\gamma \widetilde{Z}
$$

with $\gamma \neq 0$. 
These prices have

$$
\begin{aligned}
\alpha & =(1-\beta) m_{x}-\left(\beta \sigma_{\varepsilon}^{2}-(1-\beta) \sigma_{y}^{2}\right) \frac{(1-\beta) \sigma_{y}^{2} \ell}{(1-k)(1-\beta) \sigma_{y}^{2}+k \beta \sigma_{\varepsilon}^{2}} \\
\gamma & =\beta \sigma_{\varepsilon}^{2} \frac{(1-\beta) \sigma_{y}^{2}}{(1-k)(1-\beta) \sigma_{y}^{2}+k \beta \sigma_{\varepsilon}^{2}}
\end{aligned}
$$

for two possible $\beta$,

$$
\begin{aligned}
& \beta^{-}=\frac{\sigma_{y}^{4}(1-k)^{2}+\left(k^{2}-k\right) \sigma_{y}^{2} \sigma_{\varepsilon}^{2}+\frac{1}{2} \sigma_{y}^{2} \sigma_{z}^{2} \sigma_{\varepsilon}^{4}+\sigma_{y}^{4} \sigma_{z}^{2} \sigma_{\varepsilon}^{2}-\frac{1}{2} \sqrt{\sigma_{y}^{4} \sigma_{z}^{4} \sigma_{\varepsilon}^{8}-4 k \sigma_{y}^{4} \sigma_{z}^{2} \sigma_{\varepsilon}^{6}}}{\sigma_{y}^{4}(1-k)^{2}+\left(k^{2}-k\right) \sigma_{y}^{2} \sigma_{\varepsilon}^{2}+k^{2} \sigma_{\varepsilon}^{4}+\sigma_{y}^{2} \sigma_{z}^{2} \sigma_{\varepsilon}^{4}+\sigma_{y}^{4} \sigma_{z}^{2} \sigma_{\varepsilon}^{2}} \\
& \beta^{+}=\frac{\sigma_{y}^{4}(1-k)^{2}+\left(k^{2}-k\right) \sigma_{y}^{2} \sigma_{\varepsilon}^{2}+\frac{1}{2} \sigma_{y}^{2} \sigma_{z}^{2} \sigma_{\varepsilon}^{4}+\sigma_{y}^{4} \sigma_{z}^{2} \sigma_{\varepsilon}^{2}+\frac{1}{2} \sqrt{\sigma_{y}^{4} \sigma_{z}^{4} \sigma_{\varepsilon}^{8}-4 k \sigma_{y}^{4} \sigma_{z}^{2} \sigma_{\varepsilon}^{6}}}{\sigma_{y}^{4}(1-k)^{2}+2\left(k^{2}-k\right) \sigma_{y}^{2} \sigma_{\varepsilon}^{2}+k^{2} \sigma_{\varepsilon}^{4}+\sigma_{y}^{2} \sigma_{z}^{2} \sigma_{\varepsilon}^{4}+\sigma_{y}^{4} \sigma_{z}^{2} \sigma_{\varepsilon}^{2}} .
\end{aligned}
$$

In fact, the cases $\sigma_{u}=0$ and $\sigma_{u}>0$ lead to the same third degree polynomial for $\beta$. When $\sigma_{u}$ is positive, this polynomial admits one or three roots in $(0,1)$. When $\sigma_{u}$ approaches 0 , one of these roots converges to 1 which leads to $\gamma=0$. This solution is then no longer admissible. We require an additional condition, namely $\sigma_{\varepsilon}^{2} \sigma_{z}^{2} \geq 4 k$, in order to ensure that the polynomial admits other roots in $(0,1)$.

When $k=0$, which corresponds to the exogenous case, $\beta^{-}$is our solution $\beta_{e}$ of Section 3.1 and $\beta^{+}=1$ corresponds to $\beta_{R}$ of Section 2 and is a degenerate solution (full revelation of $\tilde{X}$ ). More generally, we will see in the next section that only one of the two roots, namely $\beta^{-}$, has good properties and is sensible.

Having solved the equilibrium price, it is now possible to explicitly examine agents' beliefs and demand functions in equilibrium. The following are straightforward corollaries of Theorem 4 .

Corollary 4. Under the assumtions of Theorem 4, the distribution of $\widetilde{X}$ as assessed 
by agent $i$ using his private signal $\widetilde{Y}_{i}$, the equilibrium price $\widetilde{p}$ and the observation of his own risk-aversion $a_{i}$, is normal with expectation

$$
E_{i}[\tilde{X}]=B_{i}+C \widetilde{Y}_{i}+D \widetilde{p}
$$

and variance

$$
\operatorname{Var}_{i}[\tilde{X}] \equiv V=(1-\beta) \sigma_{y}^{2}
$$

with

$$
\begin{aligned}
B_{i} & =m_{x}(1-\beta)+D \gamma A_{i}-D \alpha \\
C & =\frac{(1-\beta) \sigma_{y}^{2}}{\sigma_{\varepsilon}^{2}} \\
D & =\frac{\beta \sigma_{\varepsilon}^{2}-(1-\beta) \sigma_{y}^{2}}{\beta \sigma_{\varepsilon}^{2}}
\end{aligned}
$$

where $\beta$ and $\gamma$ are the same as in Theorem 3.

Notice that $E_{i}[\widetilde{X}]$ increases with the private signal $\widetilde{Y}_{i}$, with the equilibrium price $\widetilde{p}$ and with the level of risk-aversion $a_{i}$ whereas $\operatorname{Var}_{i}[\tilde{X}]$ is independent of $a_{i}$, which means that all other things being equal, the distribution of $\widetilde{X}$ conditional to the information $I_{i}$ increases with the level of risk-aversion in the sense of the first stochastic dominance.

\subsubsection{Equilibrium selection and comparative statics}

In this section, we consider the endogenous setting with $\sigma_{u}=0$, for which we have found two possible equilibria (Theorem 3), and we argue, from different points of view, that only one of the two roots, namely $\beta^{-}$, has a "natural behaviour". This means that only one of the two possible equilibria is sensible. We start with asymptotic arguments, 
considering in particular the behaviour of both roots when the price becomes noninformative, i.e. when $\sigma_{z}^{2} \rightarrow \infty$. We then consider the behaviour of both roots with respect to different variables, in particular $\sigma_{x}^{2}, \sigma_{\varepsilon}^{2}$ and $\sigma_{z}^{2}$.

Let us analyze the behaviour of both roots $\beta^{-}$and $\beta^{+}$when the price becomes noninformative, i.e. when $\sigma_{z}^{2} \rightarrow \infty$. It is easy to obtain that $\beta^{+} \underset{\sigma_{z}^{2} \rightarrow \infty}{\rightarrow} 1$ and that $\beta^{-} \underset{\sigma_{z}^{2} \rightarrow \infty}{\rightarrow} \frac{\sigma_{y}^{2}}{\sigma_{\varepsilon}^{2}+\sigma_{y}^{2}}$. When $\sigma_{z}^{2} \rightarrow \infty$, the natural value is $\frac{\sigma_{y}^{2}}{\sigma_{\varepsilon}^{2}+\sigma_{y}^{2}}$. Indeed, an infinite $\sigma_{z}^{2}$ corresponds to the situation where the price becomes noninformative. The value $\frac{\sigma_{y}^{2}}{\sigma_{\varepsilon}^{2}+\sigma_{y}^{2}}$ is equal to $\beta_{W}$ and corresponds to a Walrasian equilibrium in which each agent only uses the private signal, and is therefore natural for an infinite $\sigma_{z}^{2}$, whereas the value of 1 is equal to $\beta_{R}$ and corresponds to the situation in which information is fully revealed.

Furthermore, when $\sigma_{z}^{2} \rightarrow \infty$, it is easy to obtain from Corollary 4 that the conditional expected value $E_{i}[\tilde{X}]$ converges to 0 for $\beta_{+}$and to the expected value of an agent only using the private signal given by $m_{x}\left(1-\beta_{W}\right)+\beta_{W} \widetilde{Y}_{i}$ for $\beta^{-}$.

We now consider the behaviour of both roots, or more precisely their monotonicity, with respect to different variables. We introduce the following notations. Let $u \equiv \frac{\sigma_{\varepsilon}^{2}}{\sigma_{y}^{2}}$ and $v \equiv \sigma_{y}^{2} \sigma_{z}^{2}$. We are interested in the monotonicity of $\beta$ with respect to $\sigma_{\varepsilon}^{2}$ (precision of individual information) when $\sigma_{y}^{2}$ and $\sigma_{z}^{2}$ remain constant. This amounts to computing $\frac{d \beta}{d u}$ when $v$ remains constant. We prove in the Appendix that $\frac{d}{d u} \beta^{-}<0$. This means that $\beta^{-}$decreases when the precision of individual information $1 / \sigma_{\varepsilon}^{2}$ decreases, when $\sigma_{y}^{2}$ and $\sigma_{z}^{2}$ remain constant. The converse result is obtained for $\beta^{+}$. The result obtained for $\beta^{-}$ seems natural; indeed, since the coefficient $\beta^{-}$represents the weight in the equilibrium price of $\tilde{Y}$, which is the average of all private signals, it seems natural that it would decrease when the precision of the individual signals decreases for all agents.

It is interesting to analyze the monotonicity of $\beta^{-}$with respect to $\sigma_{y}^{2}$ (total noise) 
when $\frac{\sigma_{\varepsilon}^{2}}{\sigma_{y}^{2}}$ and $\sigma_{z}^{2}$ remain constant. We compute $\frac{d \beta^{-}}{d v}$ and prove in the Appendix that $\frac{d}{d v} \beta^{-}<0$, which implies that $\beta^{-}$decreases when the total noise $\sigma_{y}^{2}$ increases (when $\frac{\sigma_{\varepsilon}^{2}}{\sigma_{y}^{2}}$ and $\sigma_{z}^{2}$ remain constant). This means that the weight of $\widetilde{X}$ in the equilibrium price, represented by $\beta^{-}$, decreases when the uncertainty about $\widetilde{X}$ increases (other things remaining equal), which seems natural. This is consistent with the result of Hellwig (1980), in which the weight of $\widetilde{X}$ in the equilibrium price also decreases with $\sigma_{y}^{2}$ when $\frac{\sigma_{\varepsilon}^{2}}{\sigma_{y}^{2}}$ remains constant. Moreover, if we want to analyze the behaviour of $\beta^{-}$with respect to $\sigma_{y}^{2}$ when $\sigma_{\varepsilon}^{2}$ and $\sigma_{z}^{2}$ remain constant, we compute $-u D_{u}+v D_{v}$. We prove in the Appendix that this quantity is positive, which means that $\beta^{-}$increases when the total noise $\sigma_{y}^{2}$ increases when $\sigma_{\varepsilon}^{2}$ and $\sigma_{z}^{2}$ remain constant. In other words, the precision effect is stronger than the noise effect. The same result, i.e. the weight of $\widetilde{X}$ in the equilibrium price increases with $\sigma_{x}^{2}$, is obtained in Diamond and Verrecchia (1981).

Furthermore, the fact that $\frac{d}{d v} \beta^{-}<0$ also means that $\beta^{-}$decreases when the total noise $\sigma_{z}^{2}$ increases when $\sigma_{\varepsilon}^{2}$ and $\sigma_{y}^{2}$ remain constant. In fact, when $\sigma_{z}^{2}$ increases, all other things being equal, the price becomes less informative and the weight of $\widetilde{X}$ in the price is then smaller (and the relative weight of $\widetilde{Z}$ is higher). The limit case is given by the Walras case that corresponds to an infinite value for $\sigma_{z}^{2}$ and to the smallest possible value for $\beta^{-}$.

The converse results are obtained for $\beta^{+}$.

We now consider the behaviour of $\gamma^{-}$. We prove in the Appendix that the coefficient $r^{-}=\frac{\gamma^{-}}{\beta^{-}}$, which represents the relative contribution of $\widetilde{Z}$ and $\widetilde{X}$ to variations in the equilibrium price, increases when the total noise $\sigma_{y}^{2}$ increases when $\frac{\sigma_{\varepsilon}^{2}}{\sigma_{y}^{2}}$ and $\sigma_{z}^{2}$ remain constant. Therefore the relative weight of the average level of risk-aversion $\widetilde{Z}$ with respect to the weight of the return of the risky asset $\tilde{X}$ increases with the uncertainty on $\tilde{X}$. 
Finally, we check for $k=1$, that $\gamma^{-}$increases with $\sigma_{\varepsilon}^{2}$ (all other things being equal),

with $\sigma_{z}^{2}$ (all other things being equal), with $\sigma_{x}^{2}$ (when $\frac{\sigma_{\varepsilon}^{2}}{\sigma_{x}^{2}}$ remain constant) and with $\sigma_{x}^{2}$ (all other things being equal).

\section{Equilibrium Properties}

In this section, we analyze the properties of the considered equilibria. We start by analyzing, in equilibrium, the natural link between pessimism/optimism and risk-aversion/risktolerance. More precisely, we show that in the model with an endogenous specification of the average level of risk-aversion, a positive correlation between optimism (resp. pessimism) and risk-aversion (resp. risk-tolerance) naturally emerges.

We then analyze the impact of this correlation on the market price of risk and on the risk sharing schemes, and how they depart from those observed in the standard model.

Finally, our model also makes it possible to analyze how the arrival of more accurate information affects the agents' utility at the individual or collective level in an economy with incomplete and differential information. Once again, it is particularly interesting to analyze how the results obtained in our setting differ from the standard ones in which only the Hirschleifer effect is at work.

\subsection{Link between individual risk-aversion and belief}

The aim of this subsection is to determine if, in our model, the interaction between agents induces a correlation between individual belief and individual risk-aversion. More precisely, having supposed that individual risk-aversion and individual prior belief (or more precisely the individual signals) are independant, we analyze the impact of the agents interaction through prices by computing the covariance between posterior belief 
(or more precisely the posterior expected return) and risk-aversion. We denote this covariance by $K$. We will denote by $K^{\prime}$ the covariance between posterior belief and risk-tolerance.

In the exogenous setting, it is immediate that there is no correlation between posterior expected return and individual risk-aversion. Indeed, in this setting we have

$$
E\left[\tilde{X} \mid I_{i}\right]=B_{e}+C_{e} y_{i}+D_{e} \beta_{e} y-D_{e} \gamma_{e} z
$$

and the covariance is then given by

$$
\begin{aligned}
\int_{0}^{1} E\left[\tilde{X} \mid I_{i}\right]\left(\frac{1}{a_{i}}-\frac{1}{z}\right) d i & =\int_{0}^{1}\left[B_{e}+C_{e} y_{i}+D_{e} \beta_{e} y-D_{e} \gamma_{e} z\right]\left(\frac{1}{a_{i}}-\frac{1}{z}\right) d i \\
& =\int_{0}^{1}\left[C_{e} y_{i}\right]\left(\frac{1}{a_{i}}-\frac{1}{z}\right) d i \\
& =0 .
\end{aligned}
$$

It is easy to check that the same result holds in the two benchmark models considered at the end of Section 2, namely the Walrasian equilibrium model and the fully-revealing rational expectations model.

In the endogenous setting, we have seen that the agent individual posterior belief increases with the level of risk-aversion in the sense of the first stochastic dominance. There is then a positive correlation between optimism and risk-aversion. In order to measure the intensity of this correlation, recall that the individual posterior expected return is given by

$$
E\left[\tilde{X} \mid I_{i}\right]=m_{x}+C\left(y_{i}-m_{x}\right)+D\left(\beta\left(y-m_{x}\right)-\gamma\left(z-A_{i}\right)\right)
$$


If we denote by $\bar{a}=\int a_{i} d i$ the average risk-aversion, the covariances $K$ and $K^{\prime}$ are given by

$$
\begin{aligned}
K & =\int_{0}^{1} E\left[\tilde{X} \mid I_{i}\right]\left(a_{i}-\bar{a}\right) d i \\
& =D \gamma \int_{0}^{1}\left(A_{i}-z\right)\left(a_{i}-\bar{a}\right) d i \\
& =D \gamma \int_{0}^{1}\left(k a_{i}-\ell-z\right)\left(a_{i}-\bar{a}\right) d i \\
& =D \gamma k \operatorname{Var}\left(a_{i}\right)>0
\end{aligned}
$$

and

$$
\begin{aligned}
K^{\prime} & =\int_{0}^{1} E\left[\tilde{X} \mid I_{i}\right]\left(\frac{1}{a_{i}}-\frac{1}{z}\right) d i \\
& =D \gamma \int_{0}^{1}\left(A_{i}-z\right)\left(\frac{1}{a_{i}}-\frac{1}{z}\right) d i \\
& =D \gamma k \int_{0}^{1}\left(a_{i}-z\right)\left(\frac{1}{a_{i}}-\frac{1}{z}\right) d i \\
& =D \gamma k\left(1-\frac{\bar{a}}{z}\right)<0 .
\end{aligned}
$$

Both covariance formulas involve the dispersion of individual risk-aversion levels. Indeed, the ratio $\frac{\bar{a}}{z}$ (where $\bar{a}$ is the arithmetic average of the individual risk-aversion levels and $z$ the harmonic average) is directly related to the dispersion.

Optimism (resp. pessimism) and risk-aversion (resp. risk-tolerance) are positively correlated. The intuition for this positive correlation is simple. In the model with an endogenous specification of the average level of risk-aversion, for a given equilibrium 
price, which, as we have seen, increases with the return of the risky asset and decreases with the average level of risk-aversion, a more risk-averse agent will perceive a higher average level of risk-aversion and, by observing a given equilibrium price, will infer a higher expected return.

Our formulas for the equilibrium price are similar to those of Diamond and Verecchia (1981) except that our random variable $\tilde{Z}$ is replaced by the total supply. In their setting the total supply is a random variable and each agent takes into account his own supply in his estimation of the total supply. In our setting, the individual posterior distribution of the average level of risk-aversion for agent $i$ increases in the sense of the first-order dominance with the risk-aversion of agent $i$. In their setting, the individual posterior total supply distribution for agent $i$ increases in the sense of the first-order dominance with the supply of agent $i$. This therefore leads to a positive correlation between individual optimism and individual supply in the setting of Diamond and Verecchia (1981). However, in their case, there is a finite number of agents and the impact of individual supply on the total supply tends to be negligible when the number of agents increases.

\subsection{Collective pessimism}

The positive correlation we have found between risk-tolerance and pessimism is particularly important since as shown by Jouini-Napp (2004) in a different setting, such a positive correlation induces pessimism at the aggregate level (even if, on average, there is no pessimism at the individual level); pessimism at the aggregate level "increases" the risk premium and "decreases" the risk free rate (Abel, 2002, Jouini-Napp, 2005), which is interesting in light of the risk premium and risk free rate puzzles.

The aim of this section is to analyze the impact of the correlation between risktolerance and pessimism on the consensus belief (i.e. the belief which, if held by all 
individuals, would lead to the same equilibrium price).

We have already seen in Equation (3.6) that

$$
\widetilde{p}=\frac{\int \frac{E_{i}(\tilde{X})}{a_{i}} d i}{\int \frac{1}{a_{i}} d i}-V z
$$

and the equilibrium price in our setting corresponds then to the equilibrium price in an economy with homogenous beliefs in which the common belief is characterized by its first two moments $\frac{\int \frac{E_{i}(\widetilde{X})}{a_{i}} d i}{\int \frac{1}{a_{i}} d i}$ and $V$. The collective pessimism/optimism is then measured by

$$
\frac{\int \frac{E_{i}(\tilde{X})}{a_{i}} d i}{\int \frac{1}{a_{i}} d i}=z K^{\prime}+\int_{0}^{1} E\left[\tilde{X} \mid I_{i}\right] d i
$$

If we consider the average collective pessimism/optimism (over all possible states of the world) we obtain

$$
\int\left(\frac{\int \frac{E_{i}(\widetilde{X})}{a_{i}} d i}{\int \frac{1}{a_{i}} d i}\right) d P_{(Y,, Z)}=m_{x}-D \gamma z(1-k)-D \gamma \ell<m_{x}
$$

The collective belief is then pessimistic even though there is no bias in the individual beliefs after the observation of the private signals. Indeed, $\int_{0}^{1} E\left[\tilde{X} \mid y_{i}\right] d i$ is equal to $m_{x}$ on average (over all possible states of the world).

\subsection{Market price of risk}

The aim of this section is to analyze the impact of the collective pessimism (or of the correlation between belief and risk-aversion) on the market price of risk.

In the setting of the two benchmark models, we easily deduce from Equations (2.3) and (2.5) that the ex-ante average price (over all the possible states of the world) is of 
the form

$$
\bar{p}=m_{x}-z V
$$

where $V$ is the ex-post variance $V\left(X \mid I_{i}\right)$.

Equation (4.9) implies that in both settings, the ex-ante "risk-premium" (measured by the spread between the average asset's payoff and the average asset's price) is given by $z V$ and the average "price of risk" is given by $z$. It then appears that the introduction of rational expectations does not modify the average price of risk.

Now let us consider the case with an exogenous specification of the average level of risk-aversion. From Equation (3.3), we obtain that the ex-ante average price in this framework is

$$
\bar{p}=m_{x}-\gamma_{e}\left(z-m_{z}\right)-V_{e} m_{z}
$$

If the exogenous distribution on $\tilde{Z}$ is unbiased, i.e. if $m_{z}=z$, the average ex-ante riskpremium is given by $V z$ and the average price of risk is given by $z$. The introduction of an exogenous noise on the average level of risk-aversion then has no impact on the average price of the risk with respect to the two considered benchmark frameworks. Note that the condition that $m_{z}=z$ is equivalent to assuming that there is no bias on average on the posterior beliefs, i.e. $\int E\left[\tilde{X} \mid I_{i}\right] d P_{\left(Y, \varepsilon_{i}, Z\right)}=m_{x}$.

The conclusions in the case with an endogenous specification of the average level of risk-aversion are quite different. From equation (3.6), we have

$$
\begin{aligned}
\bar{p} & =m_{x}-D \gamma z(1-k)-D \gamma \ell-z V \\
& =m_{x}-\frac{1}{(k-1) D+1} V z-\frac{D}{(k-1) D+1} V \ell
\end{aligned}
$$


In the case $k=1$, this formula resembles the one obtained in the Walrasian case except for our additional term $-D V \ell$ which corresponds to $K^{\prime}$. The ex-ante average price of risk is then given by $z+D \ell$. The introduction of an endogenous specification of the average level of risk-aversion then leads to an increase in the market price of the risk with respect to the benchmark cases and with respect to the exogenous specification case.

In the case $k<1$, the average market price of risk is given by $\frac{1}{(k-1) D+1}(z+D \ell)$. Since $D \leq 1$, we have $\frac{1}{(k-1) D+1} \geq 1$ and we obtain a more pronounced impact on the market price of risk.

In the case $k=1$ and when the precision of the signals is sufficiently high, i.e. when $\sigma_{\varepsilon}^{2}$ is small, the parameter $D$ is near 1 . This means that the market price of risk is around $z+\ell$ and the increase in the market price of risk with respect to the benchmark cases is measured by $\frac{z+\ell}{z}$ the ratio between the arithmetic average of the individual levels of risk aversion and the harmonic average of the individual levels of risk aversion. For instance, when the individual levels of risk aversion follow a log-normal distribution $a_{i} \sim \ln \mathcal{N}\left(\mu, \sigma^{2}\right)$, the arithmetic average is equal to $\exp \left(\mu+\frac{1}{2} \sigma^{2}\right)$, the harmonic average is equal to $\exp \left(\mu-\frac{1}{2} \sigma^{2}\right)$ and the ratio is equal to $\exp \sigma^{2}$.

The interpretation of the increase of the market price of risk in the setting with an endogenous specification of the risk-aversion is the following. Since the belief of the representative agent is an average of the individual beliefs weighted by the risktolerances, the positive correlation between pessimism and risk-tolerance induces a more pessimistic consensus belief, hence higher risk premium and market price of risk. 


\subsection{Risk-sharing schemes}

The aim of this subsection is to determine the extent to which an endogenous specification of the average level of risk-aversion modifies the risk sharing schemes.

We compute the average of the individual optimal demand over the different possible signals, that we shall denote by $M_{i}^{\text {end }}$ :

$$
\begin{aligned}
M_{i}^{\text {end }} & \equiv \int \theta_{i} d \varepsilon_{i} \\
& =\frac{D k}{(k-1) D+1}+\frac{(1-D)}{(k-1) D+1} \frac{z}{a_{i}} .
\end{aligned}
$$

We first note that, since $D \leq 1, M_{i}$ is decreasing in $a_{i}$, which is natural; a more risk-averse agent will have a lower optimal demand in the risky asset.

We now compare the risk-sharing schemes in the endogenous setting with the "reference situation" of an exogenous specification of the average level of risk-aversion. With $M_{i}^{e x o}$ denoting the average (over all possible signals) of individual optimal demands in the "reference situation", we easily obtain

$$
\begin{aligned}
M_{i}^{\text {exo }} & \equiv \int_{z} \theta_{i} d \varepsilon_{i} \\
& =\frac{z}{a_{i}} .
\end{aligned}
$$

The benchmark models lead to the same result.

There is less risk-sharing in the endogenous setting. More precisely, the impact of the dispersion of the level of risk-aversion among individuals is weakened in the endogenous setting, in the sense that the less risk-averse insure less than in the reference setting. The interpretation is simple. In the reference situation, on average over the signals, a relatively very risk-averse (resp. tolerant) individual will have very low (resp. high) 
optimal demand. In the endogenous setting, as we have seen in the previous subsection, the very risk-averse becomes more optimistic, which raises his optimal demand, and the very risk-tolerant agent becomes more pessimistic, which reduces his optimal demand. As a result, there is less dispersion in the optimal demands.

\subsection{Welfare considerations}

The aim of this section is to analyze how the arrival of more precise information affects the agents' utility at the individual or collective level in an economy with incomplete and differential information in which the distribution of risk-aversion is a source of noise. Once again, it is particularly interesting to analyze how the results obtained in our setting differ from the standard ones, in which only the Hirschleifer effect ${ }^{5}$ is at work and the arrival of more precise information typically reduces agents' welfare.

We shall define an aggregate utility function, representing the average welfare of the economy, and analyze how it reacts to an increase in the precision of the information.

In this section and for reasons of tractability, we assume that the utility function of agent $i$ is of the following mean-variance form

$$
u_{i}(w)=a_{i} E_{i}[w]-\frac{1}{2} a_{i}^{2} \operatorname{Var}_{i}[w] .
$$

\footnotetext{
${ }^{5}$ The possibility that information may have a negative value is commonly known in literature as the Hirshleifer effect. Drèze (1960) was the first to identify this phenomenon and Hirshleifer (1971) formalized Drèze's argument using a general equilibrium setting. In his example, there are two states of the world, agents are risk averse and the endowments of wealth in the two states differ across the agents. If the agents trade in complete markets for contingent claims before the state is realized, then they will share some of the risk. If, however, the agents perfectly learn the state before they trade, then there will be no trade at all; thus, from an ex ante viewpoint, under perfect information each agent simply consumes his endowment, which is Pareto inferior to the allocation of risk with no information.
} 
It leads to the same optimization program as with the exponential utility function, i.e., the optimal demand for the risky asset is given by

$$
\theta_{i}^{*}=\frac{E\left[X \mid I_{i}\right]-p}{a_{i} V}
$$

The level of utility attained by agent $i$ at the optimal demand $\theta_{i}$ is then given by

$$
\begin{aligned}
U_{i} & =a_{i} E_{i}\left[\theta_{i}(X-p)+p\right]-\frac{1}{2} a_{i}^{2} \operatorname{Var}_{i}\left[\theta_{i}(X-p)+p\right] \\
& =\frac{1}{2}\left(a+b y_{i}+c y+d A_{i}+e z\right)^{2}+a_{i}(\alpha+\beta y-\gamma z)
\end{aligned}
$$

with

$$
\left\{\begin{array}{c}
a=\frac{1}{\sqrt{V}} m_{x}(1-\beta)-\alpha \\
b=-c=\frac{C}{\sqrt{V}} \\
d=\frac{e}{1-D}=\frac{1}{\sqrt{V}} \frac{D}{(k-1) D+1}
\end{array}\right.
$$

In the endogenous setting, for each agent, the individual level of utility, at the optimal demand, can be written in the following form $U_{i}=g\left(Y, \varepsilon_{i}, Z\right)$, where $\left(Y, \varepsilon_{i}, Z\right) \sim$ $\mathcal{N}\left(m_{x}, \sigma_{x}^{2}\right) \otimes \mathcal{N}\left(0, \sigma_{\varepsilon}^{2}\right) \otimes \mathcal{N}\left(A_{i}, \sigma_{z}^{2}\right)$. Ex-ante, the average level of expected utility for agent $i$ is then

$$
\begin{aligned}
& \int g\left(y, e_{i}, z\right) d P_{\left(Y, \varepsilon_{i}, Z\right)}\left(y, e_{i}, z\right) \\
= & a_{i}\left(\alpha+\beta m_{x}-\gamma A_{i}\right)+\frac{1}{2} e^{2} \sigma_{z}^{2}+\frac{1}{2} e^{2} A_{i}^{2}+\left(a+(b+c) m_{x}+d A_{i}\right) e A_{i}+\frac{1}{2} c^{2} \sigma_{\varepsilon}^{2} \\
& +\frac{1}{2}\left(a+d A_{i}\right)^{2}+\left(a+d A_{i}\right)(b+c) m_{x}+\frac{1}{2}(b+c)^{2} \sigma_{x}^{2}+\frac{1}{2}(b+c)^{2} m_{x}^{2} \\
\equiv & h\left(A_{i}\right)
\end{aligned}
$$

Now, in order to compute an average level of utility among the agents, we need a 
distribution on the $A_{i}$, and the average level will then be given by $\int h(q) d P_{A_{i}}(q)$. We now consider the case $k=1$ and we make the assumption that $A_{i} \sim \mathcal{N}\left(z, \sigma_{z}^{2}\right)$ which means that there is no collective bias in the individual estimations of the collective risk-aversion. We have

$$
\begin{aligned}
\int h(q) d P_{A_{i}}(q)= & \frac{1}{2}(1-D)^{2} V \sigma_{z}^{2}+\frac{1}{2} \frac{V}{\sigma_{\varepsilon}^{2}}+(z+\ell)\left(m_{x}-D V \ell\right)-V z \ell \\
& +D \ell V(1-D) z-\frac{1}{2} V\left(z^{2}+\sigma_{z}^{2}\right)+\frac{1}{2} D^{2} \ell^{2} V+D^{2} V \ell z
\end{aligned}
$$

Before choosing specific values for $m_{x}, \sigma_{y}, \sigma_{z}, z$ and $\ell$, let us first remark that the derivative of the quantity under consideration with respect to $\sigma_{\varepsilon}^{2}$ is independent of $m_{x}$ and we can set $m_{x}=0$. Furthermore, in the case $k=1$ and for a small dispersion of the individual levels of risk aversion, we may check that we have

$$
\left(\int \frac{1}{a_{i}} d i\right)^{-1} \approx \frac{\bar{a}}{\left(1+\frac{\operatorname{Var}\left(a_{i}\right)}{a^{2}}\right)}
$$

Since we have $z=\bar{a}-l$ then our parameters should satisfy $z \approx\left(z+\ell-\frac{\sigma_{z}^{2}}{(z+\ell)}\right)$.

Figure 1 shows that with well chosen parameters, the aggregate utility level decreases then increases when the information precision increases.

In the endogenous setting, the correlation between pessimism and risk-aversion that we have exhibited limits risk sharing and has a negative impact on welfare. The arrival of more precise information therefore has a double impact: it weakens the adverse effect on trade (as risk-taking agents become more optimistic, they offer more insurance) and at the same time it brings about the Hirschleifer effect (agents are no longer able to insure against news that has already arrived). The first effect fosters risksharing trades 
and the second one discourages them. The first effect is predominant for large precision levels and the second one is predominant for small precision levels. Figure 2 shows that the positive effect on trade may offset the negative effect for all precision levels.

The same type of result has been obtained in Hatchondo (2006) in a rather different setting. In his model, noise takes the form of an unknown level of endowment. The structure of the model does not allow for an analytical solution and the paper only relies on numerical techniques. Note that a similar result was obtained in models with information asymmetries, like in Bhattacharya and Spiegel (1991), Rahi (1996), Marin and Rahi (2000), etc. In these models, information asymmetry generates an adverse selection effect since the less informed reduce their participation due to their informational disadvantage. In this framework, the arrival of more precise information reduces the information asymetry, hence weakening the adverse selection effect. Although different, the mechanism by which the Hirshleifer effect is counterbalanced shares similarities with the one presented in our model.

This means that in an economy with differential information, agents welfare may increase upon the receipt of more precise information. Although the result seems intuitive, most of the previous literature has focused on the case with homogeneous beliefs. In such a framework, only the Hirschleifer effect is at work, and so better information typically reduces welfare.

\section{Conclusion}

We have shown that the non observability of others level of risk-aversion leads to a positive correlation between optimism and risk-aversion. Indeed, the equilibrium price increases with the return of the risky asset and decreases with the average level of 
risk-aversion. Hence, more risk-averse agents perceive an equilibrium price that seems too high and infer from there high expectations on the asset's return. This positive correlation induces pessimism at the aggregate level; even though there is no pessimism on average in the individual beliefs/signals, the consensus belief is pessimistic since the beliefs of the more risk-tolerant agents are more heavily weighted. Pessimism at the aggregate level induces then a higher market price of risk.

Furthermore, the positive correlation between optimism and risk-aversion leads the less risk-averse to insure less than in the standard setting and induces less risk sharing among agents. This effect decreases when agents receive more precise information about the traded assets. The arrival of more precise information has then two effects: an increase of the total welfare due to an increase of the risk-sharing and a decrease of the total welfare related to the well-known Hirschleifer's effect. We show that the global effect might be in both directions.

It is interesting to note that a positive correlation between optimism and riskaversion has been empirically observed in a purely behavioral setting by Ben Mansour et al. (2006). In this last paper, the authors show that agents exhibit pessimism and that more risk-averse agents are more optimistic. 


\section{Appendix}

\section{Proof of Theorem 1}

Suppose that the equilibrium price is of the form $\widetilde{p}=\alpha_{e}+\beta_{e} \widetilde{X}-\gamma_{e} \widetilde{Z}$. Then the triple $\left(\widetilde{X}, \widetilde{Y}_{i}, \widetilde{p}\right)$ is normally distributed with a mean vector

$$
\left(m_{x}, m_{x}, \alpha_{e}+\beta m_{x}-\gamma m_{z}\right)
$$

and a variance-covariance matrix

$$
\left(\begin{array}{ccc}
\sigma_{y}^{2} & \sigma_{y}^{2} & \beta_{e} \sigma_{y}^{2} \\
\sigma_{y}^{2} & \sigma_{y}^{2}+\sigma_{\varepsilon}^{2} & \beta_{e} \sigma_{y}^{2} \\
\beta_{e} \sigma_{y}^{2} & \beta_{e} \sigma_{y}^{2} & \beta_{e}^{2} \sigma_{y}^{2}+\gamma_{e}^{2} \sigma_{z}^{2}
\end{array}\right)
$$

We easily obtain that the conditional distribution of $\tilde{X}$ given $I_{i}$ is also normal with mean of the form

$$
\begin{aligned}
E_{i}[\tilde{X}] & \equiv E\left[\tilde{X} \mid \tilde{Y}_{i}=y_{i}, \widetilde{p}=\alpha_{e}+\beta_{e} y-\gamma_{e} z\right] \\
& =m_{x}+\left(\begin{array}{cc}
\sigma_{y}^{2} & \beta_{e} \sigma_{y}^{2}
\end{array}\right)\left(\begin{array}{cc}
\sigma_{y}^{2}+\sigma_{\varepsilon}^{2} & \beta_{e} \sigma_{y}^{2} \\
\beta_{e} \sigma_{y}^{2} & \beta_{e}^{2} \sigma_{y}^{2}+\gamma_{e}^{2} \sigma_{z}^{2}
\end{array}\right)^{-1}\left(\begin{array}{c}
y_{i}-m_{x} \\
\beta_{e}\left(y-m_{x}\right)-\gamma_{e}\left(z-m_{z}\right)
\end{array}\right) \\
& =m_{x}+C_{e}\left(y_{i}-m_{x}\right)+D_{e}\left(\beta_{e}\left(y-m_{x}\right)-\gamma_{e}\left(z-m_{z}\right)\right) \\
& =B_{e}+C_{e} y_{i}+D_{e} \beta y-D_{e} \gamma_{e} z
\end{aligned}
$$


with

$$
\left\{\begin{array}{c}
C_{e}=\frac{\gamma_{e}^{2} \sigma_{z}^{2} \sigma_{y}^{2}}{\gamma_{e}^{2} \sigma_{y}^{2} \sigma_{z}^{2}+\beta_{e}^{2} \sigma_{y}^{2} \sigma_{\varepsilon}^{2}+\gamma_{e}^{2} \sigma_{z}^{2} \sigma_{\varepsilon}^{2}} \\
D_{e}=\frac{\beta_{e} \sigma_{y}^{2} \sigma_{\varepsilon}^{2}}{\gamma_{e}^{2} \sigma_{y}^{2} \sigma_{z}^{2}+\beta_{e}^{2} \sigma_{y}^{2} \sigma_{\varepsilon}^{2}+\gamma_{e}^{2} \sigma_{z}^{2} \sigma_{\varepsilon}^{2}} \\
B_{e}=m_{x}\left(1-C_{e}-D_{e} \beta_{e}\right)+\gamma_{e} D_{e} m_{z}
\end{array}\right.
$$

and

$$
\begin{aligned}
& \operatorname{Var}_{i}[\tilde{X}] \equiv \operatorname{Var}\left[\tilde{X} \mid \tilde{Y}_{i}=y_{i}, \tilde{p}=\alpha_{e}+\beta_{e} y-\gamma_{e} z\right] \\
= & \sigma_{y}^{2}-\left(\begin{array}{cc}
\sigma_{y}^{2} & \beta_{e} \sigma_{y}^{2}
\end{array}\right)\left(\begin{array}{cc}
\sigma_{y}^{2}+\sigma_{\varepsilon}^{2} & \beta_{e} \sigma_{y}^{2} \\
\beta_{e} \sigma_{y}^{2} & \beta_{e}^{2} \sigma_{y}^{2}+\gamma_{e}^{2} \sigma_{z}^{2}
\end{array}\right)^{-1}\left(\begin{array}{c}
\sigma_{y}^{2} \\
\beta_{e} \sigma_{y}^{2}
\end{array}\right) \\
= & \sigma_{y}^{2}\left(1-C_{e}-\beta_{e} D_{e}\right) \\
\equiv & V_{e}
\end{aligned}
$$

which is independent of the realizations of $\widetilde{Y}_{i}$ and $\widetilde{p}$.

As in the standard setting, since the utility functions are exponential and the conditional distribution of $\widetilde{X}$ given $I_{i}$ is normal, it is well known that the portfolio optimization problem leads to a demand function given by $\widetilde{\theta}_{i}=\frac{E_{i}[\widetilde{X}]-\widetilde{p}}{a_{i} \operatorname{Var} i[\widetilde{X}]}$, hence

$$
\tilde{\theta}_{i}=\frac{B_{e}+C_{e} y_{i}+D_{e} \beta_{e} y-D_{e} \gamma_{e} z-\alpha_{e}-\beta_{e} \tilde{X}+\gamma_{e} \widetilde{Z}}{a_{i} V_{e}}
$$

The market clearing conditions can be written as

$$
\begin{aligned}
\int_{0}^{1} \tilde{\theta}_{i} d i & =\frac{1}{V_{e}}\left[\left(B_{e}-\alpha_{e}\right) \widetilde{Z}^{-1}+C_{e} \widetilde{X} \widetilde{Z}^{-1}\right]+\frac{1}{V_{e}}\left[C_{e} \int_{0}^{1} \frac{\widetilde{\varepsilon_{i}}}{a_{i}} d i+\left(D_{e}-1\right)\left(\beta_{e} \widetilde{X} \widetilde{Z}^{-1}-\gamma_{e} \int_{0}^{1} \frac{\widetilde{Z}}{a_{i}} d i\right)\right] \\
& =1
\end{aligned}
$$


or equivalently, since $\int_{0}^{1} \frac{\widetilde{\varepsilon_{i}}}{a_{i}} d i=0$,

$$
\left(B_{e}-\alpha_{e}\right) \widetilde{Z}^{-1}+\left(C_{e}+D_{e} \beta_{e}-\beta_{e}\right) \tilde{X} \widetilde{Z}^{-1}-\left(D_{e}-1\right) \gamma_{e}=V_{e}
$$

There exists an equilibrium of the form (3.1) if and only if

$$
\left\{\begin{array}{c}
\alpha_{e}=B_{e}=m_{x}\left(1-\beta_{e}\right)+\gamma_{e} D_{e} m_{z} \\
\gamma_{e}=\frac{V_{e}}{\left(1-D_{e}\right)}=\frac{\sigma_{x}^{2}\left(1-\beta_{e}\right)}{\left(1-D_{e}\right)} \\
C_{e}+D_{e} \beta_{e}=\beta_{e}
\end{array} .\right.
$$

It is easy to verify that, under the condition $C_{e}+D_{e} \beta_{e}=\beta_{e}$, we have

$$
\left\{\begin{array}{c}
\gamma_{e}^{2}=\frac{\beta_{e}^{2}\left(1-\beta_{e}\right) \sigma_{y}^{2} \sigma_{\varepsilon}^{2}}{-\sigma_{y}^{2} \sigma_{z}^{2}+\beta_{e}\left(\sigma_{y}^{2} \sigma_{z}^{2}+\sigma_{z}^{2} \sigma_{\varepsilon}^{2}\right)} \\
C_{e}=\left(1-\beta_{e}\right) \sigma_{\varepsilon}^{-2} \sigma_{y}^{2} \\
D_{e}=\left(\beta_{e} \sigma_{y}^{2}-\sigma_{y}^{2}+\beta_{e} \sigma_{\varepsilon}^{2}\right) \sigma_{\varepsilon}^{-2} \beta_{e}^{-1}
\end{array}\right.
$$

and

$$
\begin{aligned}
\gamma_{e} & =\frac{\sigma_{x}^{2}\left(1-\beta_{e}\right)}{\left(1-D_{e}\right)} \Leftrightarrow \frac{\beta_{e}^{2}\left(1-\beta_{e}\right) \sigma_{y}^{2} \sigma_{\varepsilon}^{2}}{-\sigma_{y}^{2} \sigma_{z}^{2}+\beta_{e}\left(\sigma_{y}^{2} \sigma_{z}^{2}+\sigma_{z}^{2} \sigma_{\varepsilon}^{2}\right)}=\beta_{e}^{2} \sigma_{\varepsilon}^{4} \\
& \Leftrightarrow \beta_{e}=\frac{\sigma_{x}^{2}+\sigma_{x}^{2} \sigma_{\varepsilon}^{2} \sigma_{z}^{2}}{\sigma_{x}^{2}+\sigma_{x}^{2} \sigma_{\varepsilon}^{2} \sigma_{z}^{2}+\sigma_{\varepsilon}^{4} \sigma_{z}^{2}}
\end{aligned}
$$

Hence the unique solution of the previous system is

$$
\left\{\begin{array}{c}
\alpha_{e}=m_{x}\left(1-\beta_{e}\right)+m_{z}\left(\sigma_{\varepsilon}^{2} \beta_{e}-\sigma_{x}^{2}\left(1-\beta_{e}\right)\right) \\
\beta_{e}=\frac{\sigma_{x}^{2}+\sigma_{x}^{2} \sigma_{\sigma_{z}^{2}}^{2} \sigma_{z}^{2}}{\sigma_{x}^{2}+\sigma_{x}^{2} \sigma_{\varepsilon}^{2} \sigma_{z}^{2}+\sigma_{\varepsilon}^{4} \sigma_{z}^{2}} \\
\gamma_{e}=\beta_{e} \sigma_{\varepsilon}^{2}
\end{array} .\right.
$$




\section{Proof of Theorem 2}

Note first that the conditional distribution of $\widetilde{X}$ given $\widetilde{Y}_{i}, \alpha+\beta \widetilde{Y}-\gamma \widetilde{Z}$ and $a_{i}$ is the same as the conditional distribution of $\widetilde{X}$ given $\widetilde{Y}_{i}$ and $\widetilde{p} \equiv \alpha+\beta \widetilde{Y}-\gamma \widetilde{Z}$ with $\widetilde{Z} \sim \mathcal{N}\left(A_{i}, \sigma_{z}^{2}\right)$. Since the triple $\left(\widetilde{X}, \tilde{Y}_{i}, \widetilde{p}\right)$ is normally distributed with a mean vector

$$
\left(m_{x}, m_{x}, \alpha+\beta m_{x}-\gamma A_{i}\right)
$$

and a variance-covariance matrix

$$
\left(\begin{array}{ccc}
\sigma_{y}^{2}+\sigma_{u}^{2} & \sigma_{y}^{2} & \beta \sigma_{y}^{2} \\
\sigma_{y}^{2} & \sigma_{y}^{2}+\sigma_{\varepsilon}^{2} & \beta \sigma_{y}^{2} \\
\beta \sigma_{y}^{2} & \beta \sigma_{y}^{2} & \beta^{2} \sigma_{y}^{2}+\gamma^{2} \sigma_{z}^{2}
\end{array}\right)
$$

we easily obtain that the conditional distribution of $\widetilde{X}$ given $I_{i}$ is also normal with mean of the form

$$
\begin{aligned}
& E\left[\tilde{X} \mid \tilde{Y}_{i}=y_{i}, \tilde{p}=\alpha+\beta y-\gamma z\right] \\
= & m_{x}+\left(\begin{array}{cc}
\sigma_{y}^{2} & \beta \sigma_{y}^{2}
\end{array}\right)\left(\begin{array}{cc}
\sigma_{y}^{2}+\sigma_{\varepsilon}^{2} & \beta \sigma_{y}^{2} \\
\beta \sigma_{y}^{2} & \beta^{2} \sigma_{y}^{2}+\gamma^{2} \sigma_{z}^{2}
\end{array}\right){ }^{-1}\left(\begin{array}{c}
y_{i}-m_{x} \\
\beta\left(y-m_{x}\right)-\gamma\left(z-A_{i}\right)
\end{array}\right) \\
= & m_{x}+C\left(y_{i}-m_{x}\right)+D\left(\beta\left(y-m_{x}\right)-\gamma\left(z-A_{i}\right)\right)
\end{aligned}
$$


and

$$
\begin{aligned}
& \operatorname{Var}\left[\tilde{X} \mid \tilde{Y}_{i}=y_{i}, \widetilde{p}=\alpha+\beta y-\gamma z\right] \\
= & \sigma_{y}^{2}+\sigma_{u}^{2}-\left(\begin{array}{cc}
\sigma_{y}^{2} & \beta \sigma_{y}^{2}
\end{array}\right)\left(\begin{array}{cc}
\sigma_{y}^{2}+\sigma_{\varepsilon}^{2} & \beta \sigma_{y}^{2} \\
\beta \sigma_{y}^{2} & \beta^{2} \sigma_{y}^{2}+\gamma^{2} \sigma_{z}^{2}
\end{array}\right)^{-1}\left(\begin{array}{c}
\sigma_{y}^{2} \\
\beta \sigma_{y}^{2}
\end{array}\right) \\
= & \sigma_{y}^{2}(1-C-\beta D)+\sigma_{u}^{2} \\
\equiv & V
\end{aligned}
$$

which is independent of the realizations of $\widetilde{Y}_{i}$ and $\widetilde{p}$, with

$$
\begin{aligned}
(C, D) & =\left(\begin{array}{cc}
\sigma_{y}^{2} & \beta \sigma_{y}^{2}
\end{array}\right)\left(\begin{array}{cc}
\sigma_{y}^{2}+\sigma_{\varepsilon}^{2} & \beta \sigma_{y}^{2} \\
\beta \sigma_{y}^{2} & \beta^{2} \sigma_{y}^{2}+\gamma^{2} \sigma_{z}^{2}
\end{array}\right)^{-1} \\
& =\left(\frac{\gamma^{2} \sigma_{z}^{2} \sigma_{y}^{2}}{\gamma^{2} \sigma_{y}^{2} \sigma_{z}^{2}+\beta^{2} \sigma_{y}^{2} \sigma_{\varepsilon}^{2}+\gamma^{2} \sigma_{z}^{2} \sigma_{\varepsilon}^{2}}, \frac{\beta \sigma_{y}^{2} \sigma_{\varepsilon}^{2}}{\gamma^{2} \sigma_{y}^{2} \sigma_{z}^{2}+\beta^{2} \sigma_{y}^{2} \sigma_{\varepsilon}^{2}+\gamma^{2} \sigma_{z}^{2} \sigma_{\varepsilon}^{2}}\right) .
\end{aligned}
$$

As in the standard setting, since the utility functions are exponential and the conditional distribution of $\widetilde{X}$ given $I_{i}$ is normal, it is well known that the portfolio optimization problem leads to a demand function given by $\widetilde{\theta}_{i}=\frac{E_{i}[\widetilde{X}]-\widetilde{p}}{a_{i} \operatorname{Var}_{i}[\widetilde{X}]}$. This gives

$$
\begin{aligned}
\theta_{i} & =\frac{E\left[\tilde{X} \mid \tilde{Y}_{i}=y_{i}, \widetilde{p}=\alpha+\beta y-\gamma z\right]-\widetilde{p}}{a_{i} \operatorname{Var}\left[\tilde{X} \mid \tilde{Y}_{i}=y_{i}, \widetilde{p}=\alpha+\beta y-\gamma z\right]} \\
& =\frac{m_{x}+C\left(y_{i}-m_{x}\right)+D\left(\beta\left(y-m_{x}\right)-\gamma\left(z-A_{i}\right)\right)-(\alpha+\beta y-\gamma z)}{a_{i} V}
\end{aligned}
$$

Since $A_{i}=k a_{i}-\ell$ and $\int_{0}^{1} \frac{\widetilde{\varepsilon_{i}}}{a_{i}} d i=0$, we have

$$
\int_{0}^{1} \theta_{i} d i=\frac{1}{V}\left[\left(m_{x}(1-C-D \beta)-\alpha-D \gamma \ell\right) Z^{-1}+((k-1) D+1) \gamma+(C+D \beta-\beta) Y Z^{-1}\right]
$$


and there exists an equilibrium of the form (3.4) if and only if

$$
\left\{\begin{array}{c}
\alpha=m_{x}(1-\beta)-D \gamma \ell \\
\gamma=\frac{1}{(k-1) D+1} V \\
C+D \beta=\beta
\end{array}\right.
$$

or

$$
\left\{\begin{array}{c}
\alpha=m_{x}(1-\beta)-D \gamma \ell \\
\gamma=\frac{1}{(k-1) D+1} \sigma_{y}^{2}(1-C-\beta D)+\sigma_{u}^{2} \\
C+D \beta=\beta
\end{array} .\right.
$$

Since $C$ and $D$ are functions of $\beta$ and $\gamma$, it is easy to see that the third line of the system can be written in the form $f(\beta, \gamma)=0$. Analogously, the second line of the system can be written $g(\beta, \gamma)=0$, and there exists an equilibrium of the form (3.4) if and only if there exists a couple $(\beta, \gamma)$ satisfying $f(\beta, \gamma)=0$ and $g(\beta, \gamma)=0$. Now, for $\sigma_{y} \sigma_{\varepsilon}>0$

$$
f(\beta, \gamma)=0 \Leftrightarrow \gamma^{2}=\frac{\beta^{2}(1-\beta) \sigma_{y}^{2} \sigma_{\varepsilon}^{2}}{\sigma_{y}^{2} \sigma_{z}^{2}(\beta-1)+\beta \sigma_{z}^{2} \sigma_{\varepsilon}^{2}} .
$$

Then

$$
\begin{aligned}
C & =\frac{(1-\beta) \sigma_{y}^{2}}{\sigma_{\varepsilon}^{2}} \\
D & =\frac{(\beta-1) \sigma_{y}^{2}+\beta \sigma_{\varepsilon}^{2}}{\beta \sigma_{\varepsilon}^{2}}
\end{aligned}
$$

and

$$
g(\beta, \gamma)=0 \Leftrightarrow \gamma=\frac{\beta \sigma_{\varepsilon}^{2}\left(\sigma_{u}^{2}+\sigma_{y}^{2}-\beta \sigma_{y}^{2}\right)}{\sigma_{y}^{2}(1-k-\beta+k \beta)+k \beta \sigma_{\varepsilon}^{2}}
$$


There is a couple $(\beta, \gamma)$ satisfying $f(\beta, \gamma)=0$ and $g(\beta, \gamma)=0$ if and only if

$$
\frac{\beta^{2} \sigma_{\varepsilon}^{4}\left(\sigma_{u}^{2}+\sigma_{y}^{2}-\beta \sigma_{y}^{2}\right)^{2}}{\left(\sigma_{y}^{2}(1-k-\beta+k \beta)+k \beta \sigma_{\varepsilon}^{2}\right)^{2}}-\frac{\beta^{2}(1-\beta) \sigma_{y}^{2} \sigma_{\varepsilon}^{2}}{\sigma_{y}^{2} \sigma_{z}^{2}(\beta-1)+\beta \sigma_{z}^{2} \sigma_{\varepsilon}^{2}}=0
$$

or equivalently if and only if

$$
\frac{\beta^{2} \sigma_{\varepsilon}^{2}\left(A_{3} \beta^{3}+A_{2} \beta^{2}+A_{1} \beta+A_{0}\right)}{\sigma_{z}^{2}\left(\sigma_{y}^{2}-k \sigma_{y}^{2}-\beta \sigma_{y}^{2}+k \beta \sigma_{y}^{2}+k \beta \sigma_{\varepsilon}^{2}\right)^{2}\left((\beta-1) \sigma_{y}^{2}+\beta \sigma_{\varepsilon}^{2}\right)}=0
$$

with

$$
\begin{aligned}
A_{3}= & \sigma_{y}^{6}-2 k \sigma_{y}^{6}+k^{2} \sigma_{y}^{6}-2 k \sigma_{y}^{4} \sigma_{\varepsilon}^{2}+k^{2} \sigma_{y}^{2} \sigma_{\varepsilon}^{4}+2 k^{2} \sigma_{y}^{4} \sigma_{\varepsilon}^{2}+\sigma_{y}^{4} \sigma_{z}^{2} \sigma_{\varepsilon}^{4}+\sigma_{y}^{6} \sigma_{z}^{2} \sigma_{\varepsilon}^{2}, \\
A_{2}= & 6 k \sigma_{y}^{6}-3 \sigma_{y}^{6}-3 k^{2} \sigma_{y}^{6}+4 k \sigma_{y}^{4} \sigma_{\varepsilon}^{2}-k^{2} \sigma_{y}^{2} \sigma_{\varepsilon}^{4}-4 k^{2} \sigma_{y}^{4} \sigma_{\varepsilon}^{2} \\
& -2 \sigma_{y}^{4} \sigma_{z}^{2} \sigma_{\varepsilon}^{4}-3 \sigma_{y}^{6} \sigma_{z}^{2} \sigma_{\varepsilon}^{2}-2 \sigma_{u}^{2} \sigma_{y}^{2} \sigma_{z}^{2} \sigma_{\varepsilon}^{4}-2 \sigma_{u}^{2} \sigma_{y}^{4} \sigma_{z}^{2} \sigma_{\varepsilon}^{2} \\
A_{1}= & 3 \sigma_{y}^{6}-6 k \sigma_{y}^{6}+3 k^{2} \sigma_{y}^{6}-2 k \sigma_{y}^{4} \sigma_{\varepsilon}^{2}+2 k^{2} \sigma_{y}^{4} \sigma_{\varepsilon}^{2}+\sigma_{u}^{4} \sigma_{z}^{2} \sigma_{\varepsilon}^{4}+\sigma_{y}^{4} \sigma_{z}^{2} \sigma_{\varepsilon}^{4} \\
& +3 \sigma_{y}^{6} \sigma_{z}^{2} \sigma_{\varepsilon}^{2}+2 \sigma_{u}^{2} \sigma_{y}^{2} \sigma_{z}^{2} \sigma_{\varepsilon}^{4}+4 \sigma_{u}^{2} \sigma_{y}^{4} \sigma_{z}^{2} \sigma_{\varepsilon}^{2}+\sigma_{u}^{4} \sigma_{y}^{2} \sigma_{z}^{2} \sigma_{\varepsilon}^{2} \\
A_{0}= & 2 k \sigma_{y}^{6}-\sigma_{y}^{6}-k^{2} \sigma_{y}^{6}-\sigma_{y}^{6} \sigma_{z}^{2} \sigma_{\varepsilon}^{2}-2 \sigma_{u}^{2} \sigma_{y}^{4} \sigma_{z}^{2} \sigma_{\varepsilon}^{2}-\sigma_{u}^{4} \sigma_{y}^{2} \sigma_{z}^{2} \sigma_{\varepsilon}^{2}
\end{aligned}
$$

or equivalently if the following polynomial cancels

$$
P(\beta)=A_{3} \beta^{3}+A_{2} \beta^{2}+A_{1} \beta+A_{0} .
$$


Now, since

$$
\begin{aligned}
P(0) & =2 k \sigma_{y}^{6}-\sigma_{y}^{6}-k^{2} \sigma_{y}^{6}-\sigma_{y}^{6} \sigma_{z}^{2} \sigma_{\varepsilon}^{2}-2 \sigma_{u}^{2} \sigma_{y}^{4} \sigma_{z}^{2} \sigma_{\varepsilon}^{2}-\sigma_{u}^{4} \sigma_{y}^{2} \sigma_{z}^{2} \sigma_{\varepsilon}^{2} \\
& =-\sigma_{y}^{2}\left(\sigma_{y}^{4}-2 k \sigma_{y}^{4}+k^{2} \sigma_{y}^{4}+\sigma_{u}^{4} \sigma_{z}^{2} \sigma_{\varepsilon}^{2}+\sigma_{y}^{4} \sigma_{z}^{2} \sigma_{\varepsilon}^{2}+2 \sigma_{u}^{2} \sigma_{y}^{2} \sigma_{z}^{2} \sigma_{\varepsilon}^{2}\right) \\
& <0
\end{aligned}
$$

and

$$
P(1)=\sigma_{u}^{4} \sigma_{z}^{2} \sigma_{\varepsilon}^{4}>0
$$

there exists at least one root for this polynomial between 0 and 1.

\section{Proof of Theorem 3}

As seen in the proof of Theorem 2, there exists an equilibrium of the desired form when $\sigma_{u}=0$ if and only if $P(\beta)=\sigma_{y}^{2}(\beta-1) Q(\beta)=0$

with

$$
Q(\beta)=B_{2} \beta^{2}+B_{1} \beta+B_{0}
$$

for

$$
\begin{aligned}
& B_{2}=(1-k)^{2} \sigma_{y}^{4}+k^{2} \sigma_{\varepsilon}^{4}-2 k \sigma_{y}^{2} \sigma_{\varepsilon}^{2}+2 k^{2} \sigma_{y}^{2} \sigma_{\varepsilon}^{2}+\sigma_{y}^{2} \sigma_{z}^{2} \sigma_{\varepsilon}^{4}+\sigma_{y}^{4} \sigma_{z}^{2} \sigma_{\varepsilon}^{2}, \\
& B_{1}=\sigma_{y}^{2}\left(-2(1-k)^{2} \sigma_{y}^{2}+2 k \sigma_{\varepsilon}^{2}-2 k^{2} \sigma_{\varepsilon}^{2}-\sigma_{z}^{2} \sigma_{\varepsilon}^{4}-2 \sigma_{y}^{2} \sigma_{z}^{2} \sigma_{\varepsilon}^{2}\right), \\
& B_{0}=\sigma_{y}^{4}\left((1-k)^{2}+\sigma_{z}^{2} \sigma_{\varepsilon}^{2}\right) .
\end{aligned}
$$

Then $P(\beta)=0$ either for $\beta=1$ or for $\beta$ solution of $Q(\beta)=0$. The solution $\beta=1$ gives $D=1, C=0$ and $\gamma=0$ and is therefore excluded. We now look for the possible roots of $Q$. 
For $\sigma_{\varepsilon}^{2} \sigma_{z}^{2} \geq 4 k$, we find the following two roots,

$$
\begin{aligned}
& \beta^{-}=\frac{\sigma_{y}^{4}(1-k)^{2}+\left(k^{2}-k\right) \sigma_{y}^{2} \sigma_{\varepsilon}^{2}+\frac{1}{2} \sigma_{y}^{2} \sigma_{z}^{2} \sigma_{\varepsilon}^{4}+\sigma_{y}^{4} \sigma_{z}^{2} \sigma_{\varepsilon}^{2}-\frac{1}{2} \sqrt{\sigma_{y}^{4} \sigma_{z}^{4} \sigma_{\varepsilon}^{8}-4 k \sigma_{y}^{4} \sigma_{z}^{2} \sigma_{\varepsilon}^{6}}}{\sigma_{y}^{4}(1-k)^{2}+\left(k^{2}-k\right) \sigma_{y}^{2} \sigma_{\varepsilon}^{2}+k^{2} \sigma_{\varepsilon}^{4}+\sigma_{y}^{2} \sigma_{z}^{2} \sigma_{\varepsilon}^{4}+\sigma_{y}^{4} \sigma_{z}^{2} \sigma_{\varepsilon}^{2}} \\
& \beta^{+}=\frac{\sigma_{y}^{4}(1-k)^{2}+\left(k^{2}-k\right) \sigma_{y}^{2} \sigma_{\varepsilon}^{2}+\frac{1}{2} \sigma_{y}^{2} \sigma_{z}^{2} \sigma_{\varepsilon}^{4}+\sigma_{y}^{4} \sigma_{z}^{2} \sigma_{\varepsilon}^{2}+\frac{1}{2} \sqrt{\sigma_{y}^{4} \sigma_{z}^{4} \sigma_{\varepsilon}^{8}-4 k \sigma_{y}^{4} \sigma_{z}^{2} \sigma_{\varepsilon}^{6}}}{\sigma_{y}^{4}(1-k)^{2}+2\left(k^{2}-k\right) \sigma_{y}^{2} \sigma_{\varepsilon}^{2}+k^{2} \sigma_{\varepsilon}^{4}+\sigma_{y}^{2} \sigma_{z}^{2} \sigma_{\varepsilon}^{4}+\sigma_{y}^{4} \sigma_{z}^{2} \sigma_{\varepsilon}^{2}} .
\end{aligned}
$$

Since $Q(0)=B_{0}=\sigma_{y}^{4}\left((1-k)^{2}+\sigma_{z}^{2} \sigma_{\varepsilon}^{2}\right)>0$ and $Q(1)=B_{0}+B_{1}+B_{2}=\sigma_{\varepsilon}^{4} k^{2}$ are positive, either both roots belong to $(0,1)$ or they are both outside $(0,1)$. Let us prove that they are in $(0,1)$. It is easy to verify that $B_{1}$ is negative and that $B_{2}$ is positive. Indeed, $B_{1}=-\sigma_{y}^{2}\left(2(1-k)^{2} \sigma_{y}^{2}+2 k^{2} \sigma_{\varepsilon}^{2}+\sigma_{z}^{2} \sigma_{\varepsilon}^{4}+2 \sigma_{y}^{2} \sigma_{z}^{2} \sigma_{\varepsilon}^{2}\right)-\sigma_{y}^{2} \sigma_{z}^{2} \sigma_{\varepsilon}^{4}+2 k \sigma_{\varepsilon}^{2} \sigma_{y}^{2}$ which is negative since $\sigma_{z}^{2} \sigma_{\varepsilon}^{2}-4 k>0$. The quantity $B_{2}$ satisfies

$$
\begin{aligned}
B_{2} & =\sigma_{y}^{4}-2 k \sigma_{y}^{4}+k^{2} \sigma_{y}^{4}+k^{2} \sigma_{\varepsilon}^{4}-2 k \sigma_{y}^{2} \sigma_{\varepsilon}^{2}+2 k^{2} \sigma_{y}^{2} \sigma_{\varepsilon}^{2}+\sigma_{y}^{2} \sigma_{z}^{2} \sigma_{\varepsilon}^{4}+\sigma_{y}^{4} \sigma_{z}^{2} \sigma_{\varepsilon}^{2} \\
& =(k-1)^{2} \sigma_{y}^{4}+k^{2} \sigma_{\varepsilon}^{4}-2 k \sigma_{y}^{2} \sigma_{\varepsilon}^{2}+2 k^{2} \sigma_{y}^{2} \sigma_{\varepsilon}^{2}+\sigma_{y}^{2} \sigma_{z}^{2} \sigma_{\varepsilon}^{4}+\sigma_{y}^{4} \sigma_{z}^{2} \sigma_{\varepsilon}^{2} \\
& >(k-1)^{2} \sigma_{y}^{4}+k^{2} \sigma_{\varepsilon}^{4}-2 k \sigma_{y}^{2} \sigma_{\varepsilon}^{2}+2 k^{2} \sigma_{y}^{2} \sigma_{\varepsilon}^{2}+4 k \sigma_{y}^{2} \sigma_{\varepsilon}^{2}+4 k \sigma_{y}^{4} \\
& >(k-1)^{2} \sigma_{y}^{4}+k^{2} \sigma_{\varepsilon}^{4}+2 k^{2} \sigma_{y}^{2} \sigma_{\varepsilon}^{2}+2 k \sigma_{y}^{2} \sigma_{\varepsilon}^{2}+4 k \sigma_{y}^{4}
\end{aligned}
$$

hence is positive.

This implies that $-B_{1}>\sqrt{B_{1}^{2}-4 B_{0} B_{2}}$ and $\beta^{-}$, which is given by $\frac{-B_{1}-\sqrt{B_{1}^{2}-4 B_{0} B_{2}}}{2 B_{2}}$, is positive. Since the product of both roots $\frac{B_{0}}{B_{2}}$ is positive, $\beta^{+}$is also positive. Now, $\beta^{-}<1$ if and only if $\beta^{+}<1$, or equivalently if and only if the average of the roots 
$-\frac{B_{1}}{2 B_{2}}<1$ or equivalently (since $B_{2}>0$ ) if and only if $-B_{1}-2 B_{2}<0$. Now,

$$
\begin{aligned}
B_{1}+2 B_{2} & =2 k^{2} \sigma_{\varepsilon}^{4}-2 k \sigma_{y}^{2} \sigma_{\varepsilon}^{2}+2 k^{2} \sigma_{y}^{2} \sigma_{\varepsilon}^{2}+\sigma_{y}^{2} \sigma_{z}^{2} \sigma_{\varepsilon}^{4} \\
& >2 k^{2} \sigma_{\varepsilon}^{4}+2 k \sigma_{y}^{2} \sigma_{\varepsilon}^{2}+2 k^{2} \sigma_{y}^{2} \sigma_{\varepsilon}^{2} \\
& >0
\end{aligned}
$$

and $\beta^{-}$and $\beta^{+}$both belong to $(0,1)$.

\section{Proof of Corollary 4}

Immediate using the proof of Theorems (2) and (3).

Proof of $\frac{d}{d u} \beta^{-}<0$.

We have $\beta=\frac{(1-k)^{2}-k u+k^{2} u+\frac{1}{2} u^{2} v+u v-\frac{1}{2} u \sqrt{u^{2} v^{2}-4 k u v}}{(1-k)^{2}+k^{2} u^{2}-2 k u+2 k^{2} u+u^{2} v+u v}$, hence

$$
D_{u} \beta=-\frac{\left(E_{1}+E_{2} \sqrt{u^{2} v^{2}-4 k u v}\right)}{2 \sqrt{u^{2} v^{2}-4 k u v}\left(u v-2 k u-2 k+k^{2}+2 k^{2} u+u^{2} v+k^{2} u^{2}+1\right)^{2}}
$$

with

$$
\begin{aligned}
E_{1}= & v u\left(2 u v-6 k-6 k u v+12 k^{2}-6 k^{3}+4 k^{2} u-4 k^{3} u+2 k^{2} u v+2 k^{3} u^{2}+u^{2} v^{2}+2 k^{2} u^{2} v\right) \\
E_{2}= & -2 k+2 u v-4 k u v+6 k^{2}-6 k^{3}+2 k^{4}+4 k^{2} u-8 k^{3} u+4 k^{4} u+2 k^{2} u v \\
& -2 k^{3} u^{2}+2 k^{4} u^{2}+u^{2} v^{2}+2 k^{2} u^{2} v .
\end{aligned}
$$

The sign of $D_{u} \beta$ is then the opposite of the sign of $E_{1}+E_{2} \sqrt{u^{2} v^{2}-4 k u v}$. It remains to prove that this quantity is positive. We show that $\left(E_{1}\right)^{2}-\left(E_{2} \sqrt{u^{2} v^{2}-4 k u v}\right)^{2}>0$ and that $E_{1}>0$. 
We have

$$
\begin{aligned}
& E_{1}^{2}-E_{2}^{2}\left(u^{2} v^{2}-4 k u v\right) \\
= & (-4) v u k^{3}\left(8 k-2 u v+k u v-4 k^{2}-4\right)\left(u v-2 k u-2 k+k^{2}+2 k^{2} u+u^{2} v+k^{2} u^{2}+1\right)^{2}
\end{aligned}
$$

whose sign is the same as $P(k)=8 k-2 u v+k u v-4 k^{2}-4$. The roots of this polynomial,

if they exist, are given by $\frac{1}{8} u v+\frac{1}{8} \sqrt{u^{2} v^{2}-16 u v}+1$ and $\frac{1}{8} u v-\frac{1}{8} \sqrt{u^{2} v^{2}-16 u v}+1$.

Since $u v>\sqrt{u^{2} v^{2}-16 u v}$, both roots, if they exist, are greater than 1 and the sign of $P(k)$, for $k$ between 0 and 1 , is the sign of $P(0)$ and is then negative. The sign of $E_{1}^{2}-E_{2}^{2}\left(u^{2} v^{2}-4 k u v\right)$ is then positive.

The sign of $E_{1}$ is given by the sign of

$G(u, v, k)=\left(2 u v-6 k-6 k u v+12 k^{2}-6 k^{3}+4 k^{2} u-4 k^{3} u+2 k^{2} u v+2 k^{3} u^{2}+u^{2} v^{2}+2 k^{2} u^{2} v\right)$.

We have $G(u, v, 0)>0$. It remains to show that $G$ does not cancel on the domain $u v>4 k$ in order to obtain that $E_{1}$ is positive. Let $X=u v$. We have

$G(u, v, k)=X^{2}+X\left(2 k^{2}-6 k+2 k^{2} u+2\right)+12 k^{2}-6 k-6 k^{3}+4 k^{2} u-4 k^{3} u+2 k^{3} u^{2}$,

whose roots are $3 k-k^{2}-k^{2} u \pm \sqrt{k^{4}-k^{2}-2 k^{2} u-2 k^{3} u+2 k^{4} u-2 k^{3} u^{2}+k^{4} u^{2}+1}-1$.

One of the roots can be greater than $4 k$ if and only if

$$
3 k-k^{2}-k^{2} u+\sqrt{k^{4}-k^{2}-2 k^{2} u-2 k^{3} u+2 k^{4} u-2 k^{3} u^{2}+k^{4} u^{2}+1}-1>4 k
$$


or if and only if

$$
\sqrt{k^{4}-k^{2}-2 k^{2} u-2 k^{3} u+2 k^{4} u-2 k^{3} u^{2}+k^{4} u^{2}+1}>k+1+k^{2}+k^{2} u
$$

or equivalently if and only if $(-2) k(k+k u+1)^{2}>0$ which is not true.

Proof of $\frac{d}{d v} \beta^{-}<0$.

The quantity $\frac{d}{d v} \beta^{-}$can be written in the form

$$
\frac{d}{d v} \beta^{-}=\left(-\frac{1}{2}\right) \frac{(U-V)}{\left(u v-2 k u-2 k+k^{2}+2 k^{2} u+u^{2} v+k^{2} u^{2}+1\right)^{2}}
$$

with

$$
\begin{aligned}
U & =u v-2 k+4 k^{2}-2 k^{3}+4 k^{2} u-4 k^{3} u+k^{2} u v-2 k^{3} u^{2}+2 k^{2} u^{2} v+k^{2} u^{3} v, \\
V & =\sqrt{u^{2} v^{2}-4 k u v}\left(1-k^{2}-2 k^{2} u-k^{2} u^{2}\right)
\end{aligned}
$$

and $U^{2}-V^{2}=4 k^{2}\left(u v-2 k u-2 k+k^{2}+2 k^{2} u+u^{2} v+k^{2} u^{2}+1\right)^{2}>0$.

It remains to verify the sign of $U$. This quantity can be written in the form

$$
U=X\left(k^{2}+2 k^{2} u+k^{2} u^{2}+1\right)+4 k^{2}-2 k-2 k^{3}+4 k^{2} u-4 k^{3} u-2 k^{3} u^{2}
$$

There is a root such that $X>4 k$ if and only if $-\frac{4 k^{2}-2 k-2 k^{3}+4 k^{2} u-4 k^{3} u-2 k^{3} u^{2}}{k^{2}+2 k^{2} u+k^{2} u^{2}+1}>4 k$ or equivalently if and only if

$$
-4 k^{2}+2 k+2 k^{3}-4 k^{2} u+4 k^{3} u+2 k^{3} u^{2}-4 k\left(k^{2}+2 k^{2} u+k^{2} u^{2}+1\right)>0 .
$$

This quantity is equal to $(-2) k(k+k u+1)^{2}$ and is then negative. Hence $\frac{d}{d v} \beta^{-}<$ 
Proof of $-u D_{u}+v D_{v}>0$

Modulo some positive terms, the quantity $-u D_{u}+v D_{v}$ is given by

$$
-u D_{u}+v D_{v}=E_{3}-E_{4}
$$

with

$$
\begin{aligned}
E_{3}= & u v-2 k-4 k u v+6 k^{2}-6 k^{3}+2 k^{4}+4 k^{2} u-8 k^{3} u+4 k^{4} u+3 k^{2} u v-2 k^{3} u^{2} \\
& +2 k^{4} u^{2}+u^{2} v^{2}+4 k^{2} u^{2} v+k^{2} u^{3} v \\
E_{4}= & -\sqrt{u^{2} v^{2}-4 k u v}\left(1-2 k+u v+k^{2}-k^{2} u^{2}\right) .
\end{aligned}
$$

We check that

$$
E_{3}^{2}-E_{4}^{2}=4 k^{2}\left(u v-2 k+k^{2}+1\right)\left(u v-2 k u-2 k+k^{2}+2 k^{2} u+u^{2} v+k^{2} u^{2}+1\right)^{2}
$$

and the sign of $-u D_{u}+v D_{v}$ is then given by the sign of $E_{3}$.

Let $X \equiv u v, E_{3}$ is then a polynomial in $X$ whose roots are $2 k-\frac{3}{2} k^{2}-2 k^{2} u-\frac{1}{2} k^{2} u^{2} \pm$ $\frac{1}{2} \sqrt{k^{4}-2 k^{2}-8 k^{2} u+8 k^{4} u+2 k^{2} u^{2}+14 k^{4} u^{2}+8 k^{4} u^{3}+k^{4} u^{4}+1}-\frac{1}{2}$.

Let us check that both roots are such that $X-4 k<0$.

We have

$$
X-4 k=E_{5} \pm E_{6}
$$


with

$$
\begin{aligned}
& E_{5}=-2 k-\frac{3}{2} k^{2}-2 k^{2} u-\frac{1}{2} k^{2} u^{2}-\frac{1}{2} \\
& E_{6}=\frac{1}{2} \sqrt{k^{4}-2 k^{2}-8 k^{2} u+8 k^{4} u+2 k^{2} u^{2}+14 k^{4} u^{2}+8 k^{4} u^{3}+k^{4} u^{4}+1}
\end{aligned}
$$

We have $E_{5}<0$ and $E_{5}^{2}-E_{6}^{2}=2 k(k+1)(k+k u+1)^{2}>0$. Hence $X-4 k<0$.

The sign of $-u D_{u}+v D_{v}$ is then constant for $k$ in $[0,1]$ and, for $k=0$, we have $-u D_{u}+v D_{v}=u v+u^{2} v^{2}>0$.

Proof that $r^{-} \equiv \frac{\gamma^{-}}{\beta^{-}}$increases when the total noise $\sigma_{y}^{2}$ increases with $\frac{\sigma_{\varepsilon}^{2}}{\sigma_{y}^{2}}$ and $\sigma_{z}^{2}$ constants.

We have $\frac{\gamma}{\beta}=\frac{(1-\beta) \sigma_{y}^{2} \beta^{-1}}{(k-1)\left(\beta \sigma_{y}^{2}-\sigma_{y}^{2}+\beta \sigma_{\varepsilon}^{2}\right) \sigma_{\varepsilon}^{-2} \beta^{-1}+1}$ hence

$$
D_{\beta}\left(\frac{\gamma}{\beta}\right)=-\left(\sigma_{y}^{2}-k \sigma_{y}^{2}-\beta \sigma_{y}^{2}+k \beta \sigma_{y}^{2}+k \beta \sigma_{\varepsilon}^{2}\right)^{-2} \sigma_{\varepsilon}^{4} \sigma_{y}^{2} k<0
$$

Moreover, the derivative of $\frac{\gamma}{\beta}$ with respect to $X=\sigma_{y}^{2}$ is given by

$$
\begin{aligned}
& D_{X}\left(\frac{(1-\beta) X \beta^{-1}}{(k-1)\left(\beta X-X+\beta \sigma_{\varepsilon}^{2}\right) \sigma_{\varepsilon}^{-2} \beta^{-1}+1}\right) \\
= & \left(X-X k-X \beta+X k \beta+k \beta \sigma_{\varepsilon}^{2}\right)^{-2}(1-\beta) k \beta \sigma_{\varepsilon}^{4}>0
\end{aligned}
$$

and the derivative with respect to $X=\sigma_{\varepsilon}^{2}$ is given by 


$$
\begin{aligned}
& D_{X}\left(\frac{(1-\beta) \sigma_{y}^{2} \beta^{-1}}{(k-1)\left(\beta \sigma_{y}^{2}-\sigma_{y}^{2}+\beta X\right) X^{-1} \beta^{-1}+1}\right) \\
= & \left(X k \beta+\sigma_{y}^{2}-k \sigma_{y}^{2}-\beta \sigma_{y}^{2}+k \beta \sigma_{y}^{2}\right)^{-2}(1-k)(\beta-1)^{2} \sigma_{y}^{4}>0 .
\end{aligned}
$$

This implies that when $\sigma_{y}^{2}$ and $\sigma_{\varepsilon}^{2}$ increase with the precision $\frac{\sigma_{\varepsilon}^{2}}{\sigma_{y}^{2}}$ kept constant, then, as seen above, $\beta^{-}$decreases, $\sigma_{y}^{2}$ and $\sigma_{\varepsilon}^{2}$ increase, hence $\frac{\gamma}{\beta}$ increases. 


\section{References}

[1] Abel, A. B., 1989. Asset prices under heterogenous beliefs: implications for the equity premium. Unpublished working paper. University of Pennsylvania.

[2] Abel, A. B., 2002. An exploration of the effects of pessimism and doubt on asset returns. Journal of Economic Dynamics and Control, 26, 1075-1092.

[3] Admati, A. R., 1985. A noisy rational expectations equilibrium for multi asset securities markets. Econometrica, 53, 629-657.

[4] Basak, S., 2000. A model of dynamic equilibrium asset pricing with heterogenous beliefs and extraneous risk. Journal of Economic Dynamics and Control, 24, 63-95.

[5] Ben Mansour, S., Jouini, E., Marin, J.-M., Napp, C., Robert, C., 2006. Are more risk-averse agents less pessimistic? A Bayesian estimation approach. Unpublished working paper.

[6] Bhattacharya, U., Spiegel, M., 1992. Insiders, outsiders and market breakdowns. Review of Financial Studies, 4, 255-282.

[7] Brunnermeier, M. K., Parker, J. A., 2005. Optimal Expectations. American Economic Review, 95, 1092-1118.

[8] Calvet, L., Grandmont, J.-M., Lemaire, I., 2002. Aggregation of Heterogenous Beliefs and Asset Pricing in Complete Financial Markets. Unpublished working paper. .

[9] Detemple, J., Murthy, S., 1994. Intertemporal Asset Pricing with Heterogeneous Beliefs. Journal of Economic Theory, 62, 294-320. 
[10] Diamond, D. W., Verrecchia, R. E., 1981. Information aggregation in a noisy rational expectations economy. Journal of Financial Economics, 9, 221-235.

[11] Donkers, B., Melenberg, B., Van Soest, A., 2001. Estimating Risk attitudes Using Lotteries: a Large Sample Approach. Journal of Risk and Uncertainty, 22, 165-195.

[12] Drèze, J.H., 1960. Le paradoxe de l'information, Economie Appliquée, Vol. 13, 71-80.

[13] Dumas, B., Kurshev, A., Uppal, R. What can rational investors do about excessive volatility and sentiment fluctutations ? CEPR Discussion Paper $\mathrm{N}^{\circ} 5367$, available at SSRN: http://ssrn.com/abstract $=883707$

[14] Gallmeyer, M., 2000. Beliefs and volatility. Working paper, Carnegie Mellon University.

[15] Gallmeyer, M., Hollifield, B., 2002. An examination of heterogenous beliefs with a short sale constraint. Unpublished working paper. Carnegie Mellon University.

[16] Genotte, G., Leland, H., 1990. Market Liquidity, Hedging, and Crashes. American Economic Review, 80, 999-1021.

[17] Glosten, L., 1989. Insider trading, liquidity, and the role of the monopolist specialist. Journal of Business, 62, 211-235.

[18] Gollier, C., Muermann, A., 2006. Optimal Choice and Beliefs with ex-ante savoring and ex-post disappointment. Unpublished working paper. IDEI.

[19] Green, J. R., 1973. Information, efficiency and equilibrium. Discussion paper 284, Harvard Institute of Economic Research, Cambridge, MA. 
[20] Grossman, S., 1976. On the efficiency of competitive stock markets where agents have diverse information. Journal of Finance, 31, 573-585.

[21] Grossman, S., Stiglitz, J., 1981. On the impossibility of informationally efficient markets. American Economic Review, 70, 393-408.

[22] Harris, M., Raviv, A., 1993. Differences of opinion make a horse race. Review of Financial Studies, 6, 473-506.

[23] Hatchondo, J. C., 2005. The Value of Information with Heterogeneous Agents and Partially Revealing Prices. Federal Reserve Bank of Richmond Working Paper 05-6.

[24] Hellwig, M. F., 1980. On the aggregation of information in competitive markets. Journal of Economic Theory, 22, 477-498.

[25] Hirshleifer, J., 1971. The private and social value of information and the reward to inventive activity. American Economic Review, Vol. 61, 4, 561-574.

[26] Jackwerth, J. C., 2000. Recovering Risk Aversion from Option Prices and Realized Returns. Review of Financial Studies, 13(2), 433-51.

[27] Jouini, E., Napp, C., 2004. Consensus consumer and intertemporal asset pricing with heterogenous beliefs. Unpublished working paper. Université Paris-Dauphine. Available at SSRN: http://ssrn.com/abstract $=915371$.

[28] Jouini, E., Napp, C. 2006. An analysis of pessimism and doubt. Journal of Economic Dynamics and Control., 30, 1233-1260.

[29] Kyle, A. S., 1989. Informed Speculation with Imperfect Competition. Review of Economic Studies, 56, 317 - 356. 
[30] Marin, J., Rahi, R., 2000. Information revelation and market incompleteness. Review of Economic Studies, 67, 455-481.

[31] Rahi, R., 1996. Adverse selection and security design. Review of Economic Studies, $63,287-300$.

[32] Scheinkman, J. A., Xiong, W., 2003. Overconfidence and speculative bubbles. Journal of Political Economy, 111, 1183-1219.

[33] Varian, H., 1985. Divergence of Opinion in Complete Markets. Journal of Finance, 40, 309-317.

[34] Varian, H., 1989. Difference of Opinion in Financial Markets. In Financial Risk: Theory, Evidence, and Implications. Stone C.C. (Ed.), Kluwer, Dordrecht, The Netherlands.

[35] Williams, J. T., 1977. Capital Asset Prices with Heterogeneous Beliefs. Journal of Financial Economics, 5, 219-239.

[36] Zapatero, F., 1998. Effects on Financial Innovations on Market volatility when Beliefs are Heterogeneous. Journal of Economic Dynamics and Control, 22, 597626 


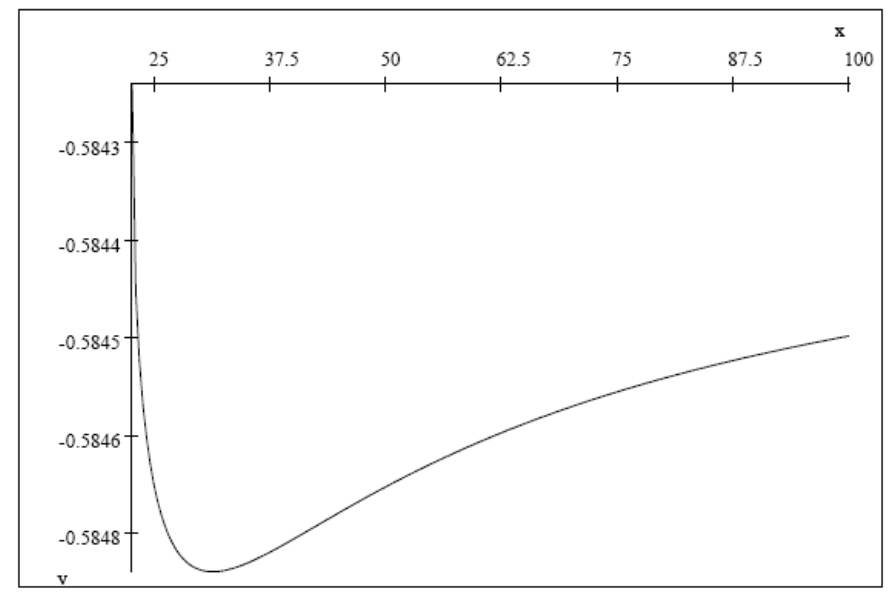

Figure 1.

This figure represents the aggregate utility as a function of $\sigma_{\varepsilon}^{2}$, the variance of the individual signals, for $\sigma_{y}=1, \sigma_{z}=0.42, z=0.95$ and $l=0.14$. When the signal precision increases, the utility first decreases (Hirschleifer effect) and then increases.

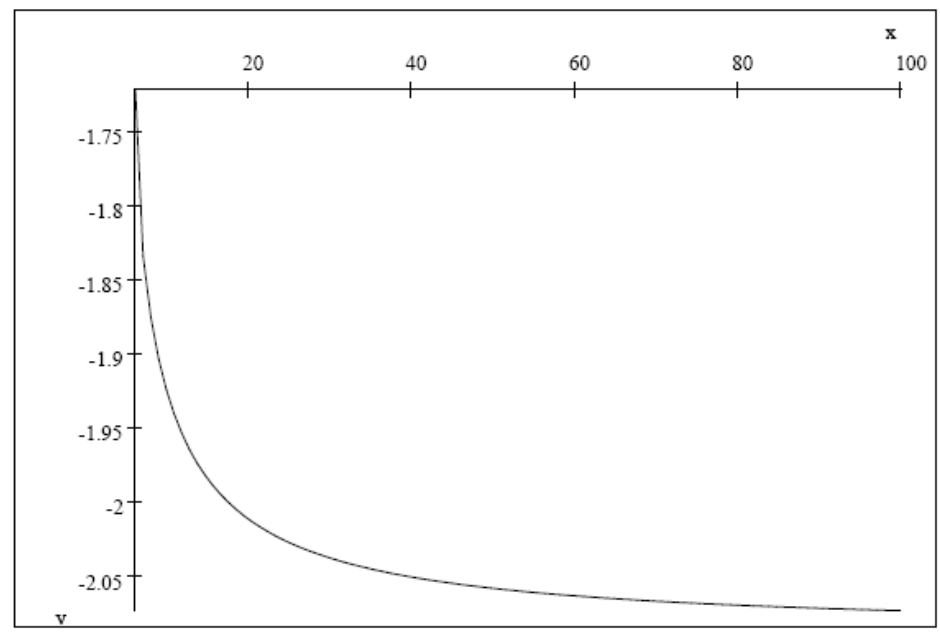

Figure 2.

This figure represents the aggregate utility as a function of $\sigma_{\varepsilon}^{2}$, the variance of the individual signals, for $\sigma_{y}=1, \sigma_{z}=0.8, z=1.8$ and $l=0.26$. When the signal precision increases, the utility increases. 